\title{
K-THEORY AND THE SINGULARITY CATEGORY OF QUOTIENT SINGULARITIES
}

\author{
NEBOJSA PAVIC AND EVGENY SHINDER
}

\begin{abstract}
In this paper we study Schlichting's K-theory groups of the Buchweitz-Orlov singularity category $\mathcal{D}^{\mathrm{sg}}(X)$ of a quasi-projective algebraic scheme $X / k$ with applications to Algebraic K-theory.

We prove for isolated quotient singularities over an algebraically closed field of characteristic zero that $\mathrm{K}_{0}\left(\mathcal{D}^{\mathrm{sg}}(X)\right)$ is finite torsion, and that $\mathrm{K}_{1}\left(\mathcal{D}^{\mathrm{sg}}(X)\right)=0$. One of the main applications is that algebraic varieties with isolated quotient singularities satisfy rational Poincaré duality on the level of the Grothendieck group; this allows computing the Grothendieck group of such varieties in terms of their resolution of singularities. Other applications concern the Grothendieck group of perfect complexes supported at a singular point and topological filtration on the Grothendieck groups.
\end{abstract}

\section{Contents}

\section{Introduction}

1. Poincaré duality for quotient singularities.

2. Cohomology and homology algebraic cycles.

3. Computing $\mathrm{K}_{0}(X$ on $\operatorname{Sing}(X))$ : the Srinivas conjecture.

4. Homological Bondal-Orlov localization conjecture.

Acknowledgements

Notation and conventions

1. Singularity K-theory

1.1. Triangulated and dg singularity categories

1.2. K-Theory of the singularity category

1.3. Topological filtration on $\mathrm{K}_{0}^{s g}$

2. Singularity K-theory of quotient singularities

2.1. The local case: non-positive K-groups

2.2. The local case: positive K-groups

2.3. The global case

2.4. Relation to the resolution of singularities

3. Examples and Applications

3.1. Torsion-free $\mathrm{K}_{0}(X)$

3.2. $A D E$ curves and threefolds

3.3. Non-vanishing $\mathrm{K}_{1}^{s g}(X)$ 
3.4. Proof of a conjecture of Srinivas for quotient singularities

\section{INTRODUCTION}

In this paper we perform a systematic study of the Schlichting K-theory groups of the dgenhancement of the Buchweitz-Orlov singularity category $\mathcal{D}^{\text {sg }}(X)=\mathcal{D}^{b}(X) / \mathcal{D}^{\text {perf }}(X)$; we call the latter K-theory groups the singularity K-theory.

Let $X / k$ be a quasi-projective scheme. Let $\mathrm{K}_{i}(X)=\mathrm{K}_{i}\left(\mathcal{D}^{\text {perf }}(X)\right)$ denote the ThomasonTrobaugh K-theory of perfect complexes, which in the quasi-projective case coincides with K-theory of vector bundles on $X$, while $\mathrm{G}_{i}(X)=\mathrm{K}_{i}\left(\mathcal{D}^{b}(X)\right)$ is Quillen's G-theory, that is

$\mathrm{K}$-theory of coherent sheaves. By construction the singularity K-theory groups $\mathrm{K}_{i}^{s g}(X)$ fit into an exact sequence

$$
\cdots \rightarrow \mathrm{K}_{i}(X) \rightarrow \mathrm{G}_{i}(X) \rightarrow \mathrm{K}_{i}^{s g}(X) \rightarrow \mathrm{K}_{i-1}(X) \rightarrow \ldots,
$$

for $i \geq 1$, finishing at

$$
\cdots \rightarrow \mathrm{K}_{1}^{s g}(X) \rightarrow \mathrm{K}_{0}(X) \rightarrow \mathrm{G}_{0}(X) \rightarrow \mathrm{K}_{0}^{s g}(X) \rightarrow 0,
$$

but negative $\mathrm{K}$ groups can be taken into account as well, see Lemma 1.10.

A classical result going back to Serre is that if $X$ is regular, then $\mathcal{D}^{\text {perf }}(X)=\mathcal{D}^{b}(X)$, so the canonical maps $\mathrm{K}_{i}(X) \rightarrow \mathrm{G}_{i}(X)$ are isomorphisms for all $i$ and $\mathrm{K}_{i}^{s g}(X)=0$. In general we may think of the singularity $\mathrm{K}$-theory groups $\mathrm{K}_{i}^{s g}(X)$ as a tool for controlling the difference between K-theory and G-theory. This approach is essentially a homological incarnation of Orlov's definition of the singularity category, and explains the terms "singularity category" and "singularity K-theory".

We develop the theory of singularity K-theory, explaining its functoriality properties and stating relevant exact sequences. Many of these properties follow directly from the work of Orlov [56, 57] once one makes sure that the relevant triangulated functors are induced from dg enhancements. For a similar perspective on studying homological invariants of the singularity category see [69, 70, 35], and for an algebraic approach to $K_{0}$ and $K_{1}$ of the singularity category via MCM modules see [41, 52, 28].

Let us motivate our study from several viewpoints, relating to earlier work in Algebraic Ktheory of singular varieties, and pointing out what singularity K-theory has to offer in each case. As a general rule our most interesting applications concentrate on isolated rational singularities, including quotient and $A D E$ singularities.

1. Poincaré duality for quotient singularities. One of the main questions which motivated this work has been the following one. If $X / k$ is a quasi-projective algebraic variety with quotient singularities, it is a natural guess that canonical maps $\mathrm{K}_{i}(X) \rightarrow \mathrm{G}_{i}(X)$ are isomorphisms up 
to torsion; indeed this could be expected as $X$ should be thought of as an analog of a $\mathbb{Q}$ manifold, while $\mathrm{K}_{i}(X) \rightarrow \mathrm{G}_{i}(X)$ may be thought as the Poincaré duality map; we use the notation $\mathrm{PD}: \mathrm{K}_{i}(X) \rightarrow \mathrm{G}_{i}(X)$ for this map. In general however it is not true that $\mathrm{PD}$ is an isomorphism up to torsion for varieties with quotient singularities.

Indeed, if either $X$ has nonisolated quotient singularities or if $i \geq 1$, then examples of Gubeladze [38] (cf Example 3.7) and Srinivas [65] (see Remark 2.21) respectively show that $\mathrm{K}_{i}(X) \rightarrow \mathrm{G}_{i}(X)$ is not an isomorphism, even after tensoring with $\mathbb{Q}$. The typical phenomenon is that $\mathrm{G}_{i}(X)$ are under control while $\mathrm{K}_{i}(X)$ become counterintuitive. In both examples of Srinivas and Gubeladze $\mathrm{K}_{i}(X) \rightarrow \mathrm{G}_{i}(X)$ has a "huge" kernel.

One of our main results is that Poincaré duality does hold up to torsion for $i=0$ in the isolated quotient singularities case:

Theorem 0.1 (See Theorem 2.23). Let $X$ be an $n$-dimensional quasi-projective variety over an algebraically closed field $k$ of characteristic zero. Assume that $X$ has isolated quotient singularities with isotropy groups $G_{i}, i=1, \ldots, m$. Then the map

$$
\mathrm{PD}: \mathrm{K}_{0}(X) \rightarrow \mathrm{G}_{0}(X)
$$

is injective, and its cokernel is a finite torsion group annihilated by $\operatorname{lcm}\left(\left|G_{1}\right|, \ldots,\left|G_{m}\right|\right)^{n-1}$.

We deduce the following Corollary of the Theorem, which often allows to conclude that $\mathrm{K}_{0}(X)$ is finitely generated:

Corollary 0.2 (See Theorem 2.28). Under the same assumptions as in the Theorem, for any resolution of singularities $\pi: Y \rightarrow X$ the pull-back $\pi^{*}: \mathrm{K}_{0}(X) \rightarrow \mathrm{K}_{0}(Y)$ is injective.

This is a strengthening of a result of Levine who proves the result in dimension up to three and shows that $\pi^{*}$ has torsion kernel in general [46].

Let us emphasize that there is in principle no easy way of controlling $\mathrm{K}_{0}(X)$ of singular varieties. To illustrate our point, let us note that it is a well-known open question in K-theory of singular varieties, whether every weighted projective space $X=\mathbb{P}\left(a_{0}, \ldots, a_{n}\right)$ has a finitely generated $\mathrm{K}_{0}(X)$ [38, Acknowledgements], [42, 5.2.3]; note that $\mathrm{G}_{0}(X)$ is finitely generated and has rank $n+1$.

One important method of computing K-theory of singular varieties has been developed in [17, 18, 19, 20, 21] and consists in relating K-theory to various sheaf cohomology groups. This method has been applied to weighted projective spaces in [50] where $\mathrm{K}_{0}(\mathbb{P}(1, \ldots, 1, a))$ has been computed (it is isomorphic to the Grothendieck group of a projective space of the same dimension, which is the answer one would expect).

Regarding weighted projective spaces, we can prove the following. If $a_{0}, \ldots, a_{n}$ are pairwise coprime, so that $X=\mathbb{P}\left(a_{0}, \ldots, a_{n}\right)$ has isolated quotient singularities, then using the Theorem and the Corollary above we deduce that $\mathrm{K}_{0}(X)$ is a free abelian group of rank $n+1$ (Application 3.2). 
The Theorem above follows using the exact sequence (0.1) once we know that for varieties with isolated quotient singularities over an algebraically closed field of characteristic zero $\mathrm{K}_{0}^{s g}(X)$ is finite torsion (Proposition 2.5) and $\mathrm{K}_{1}^{s g}(X)=0$ (Corollary 2.19). In order to study the general case we first study the local case $\mathbb{A}^{n} / G$, where a finite group $G$ acts on its linear representation. We study this local case in some detail relying on tools such as equivariant K-theory, equivariant Chow groups and cdh topology (Propositions [2.8, 2.9, 2.10, 2.18).

In contrast to the isolated quotient singularities case, the group $\mathrm{K}_{1}^{s g}(X)$ in general does not vanish for more general singularities, e.g. for rational isolated singularities (Example 3.8) or non-isolated quotient singularities (Example 3.7), and the assumption that $k$ is algebraically closed is necessary as well (Example 3.9).

2. Cohomology and homology algebraic cycles. The usual Chow groups have the functoriality property of Borel-Moore homology theory, and it has been asked by Srinivas what is the correct definition of Chow cohomology of singular varieties [66]. Taking insight from the intersection homology, it seems natural that in order to define such a theory one needs to generalize both the algebraic cycles and the rational equivalence relation. For example the Chow group $\mathrm{CH}_{\operatorname{dim}(X)-1}(X)$ (which coincides with $\mathrm{Cl}(X)$ when $X$ is normal) is the group of "homology divisors", whereas $\operatorname{Pic}(X)$ can be thought as the group of "cohomology divisors". Cohomology zero cycles have been introduced and studied in [47].

Let us now take the sheaves rather than cycles perspective, and see what we can say then. This approach is legitimate as one way to define Chow groups (up to torsion) is to take the associated graded groups for the topological filtration on $\mathrm{G}_{0}(X)$, that is the filtration given by codimension of support. See work of Gillet [33] for a realization of this approach to Chow groups and intersection theory in the regular case and Fulton [30] for the view-point on $\mathrm{K}_{0}(X) \otimes \mathbb{Q}$ as a variant for Chow-cohomology theory.

In fact the difference between homology and cohomology algebraic cycles in the singular case seems to be of the same nature as between coherent sheaves and perfect complexes. For example, if we ask the question: what makes a homology class cohomological, this can be interpreted as the question about

$$
\mathrm{K}_{0}^{s g}(X)=\operatorname{Coker}\left(\mathrm{K}_{0}(X) \rightarrow \mathrm{G}_{0}(X)\right) .
$$

We explain that there is an induced "topological" filtration by codimension of support on $\mathrm{K}_{0}^{s g}(X)$ and study its associated graded groups $\operatorname{gr}^{i} \mathrm{~K}_{0}^{s g}(X)=F^{i} \mathrm{~K}_{0}^{s g}(X) / F^{i+1} \mathrm{~K}_{0}^{s g}(X)$; we think of these groups as obstructions for the codimension $i$ homology algebraic cycles to be cohomological. This approach can be demonstrated in small dimension and codimension as follows.

Theorem 0.3. (Proposition 1.24) Let $X / k$ be a connected reduced quasi-projective scheme of pure dimension over an algebraically closed field, then (1) $\operatorname{gr}^{0} \mathrm{~K}_{0}^{s g}(X)=\mathbb{Z}^{N-1}$, where $N$ is the number of irreducible components of $X$. 
(2) If $X$ is irreducible and normal, then $\operatorname{gr}^{1} \mathrm{~K}_{0}^{s g}(X)=\mathrm{Cl}(X) / \operatorname{Pic}(X)$.

(3) $\operatorname{gr}^{\operatorname{dim} X} K_{0}^{s g}(X)=0$.

In particular, if $X$ is an irreducible normal surface, then $\mathrm{K}_{0}^{s g}(X)$ is concentrated in a single degree 1 and $\mathrm{K}_{0}^{s g}(X) \simeq \mathrm{Cl}(X) / \operatorname{Pic}(X)$.

A related and especially amusing phenomenon is that the well-known Knörrer periodicity shifts the topological filtration by one (see Proposition 1.30); this puts questions such as factoriality of threefolds and irreducibility of curves on equal footing (Application 3.5. Example 3.6).

In the case of ordinary double points of arbitrary dimension, the only nontrivial graded group for the topological filtration on $\mathrm{K}_{0}^{s g}(X)$ is in the middle codimension (Examples 1.31, 1.32). Returning to the relation between sheaves and algebraic cycles, this predicts that all cycles in codimension up to half the dimension on varieties with ordinary double points are "cohomological". For a very concrete example, note that normal varieties of dimension at least four with ordinary double points are factorial, that is $\operatorname{Pic}(X)=\mathrm{Cl}(X)$.

In the example of an isolated quotient singularity, for instance, in the local case the associated graded groups $\operatorname{gr}^{i} \mathrm{~K}_{0}^{s g}\left(\mathbb{A}^{n} / G\right)$ are typically nonzero in the range $0<i<n$ and are closely related to the $G$-equivariant Chow groups of a point [25].

3. Computing $\mathrm{K}_{0}(X$ on $\operatorname{Sing}(X))$ : the Srinivas conjecture. Let $X$ be a quasi-projective variety with isolated singularities over an algebraically closed field of characteristic zero. In [64] Srinivas introduced and studied the Grothendieck group of the exact category of coherent sheaves supported at the singular locus and having finite projective dimension (i.e. perfect as complexes); for Cohen-Macaulay isolated singularities this group is isomorphic to the Grothendieck group $\mathrm{K}_{0}(X$ on $\operatorname{Sing}(X))$ of the triangulated category of zero-dimensional perfect complexes supported at the singular points [59, Proposition 2].

There is a natural homomorphism

$$
l: \mathrm{K}_{0}(X \text { on } \operatorname{Sing}(X)) \rightarrow \mathbb{Z}^{\operatorname{Sing}(X)}
$$

induced by the length of the sheaf.

We call the question whether $l$ is an isomorphism for isolated quotient singularities the Srinivas conjecture (see [64, Page 38]). Levine has proved that $l$ is surjective for all isolated Cohen-Macaulay singularities [46, Proposition 2.6]. Furthermore, Levine proved that for isolated quotient singularities of dimension up to three in characterstic zero $l$ is an isomorphism [46, Theorem 3.3], and that it is an isomorphism up to torsion in general [46, Theorem 2.7].

On the other hand, it is known that $l$ is not always injective; for instance for a threedimensional quadric cone $x y=z w, \operatorname{Ker}(l)=\mathbb{Z} \oplus k^{*}[46$, Theorem 4.2].

Using singularity K-theory with supports we reprove surjectivity of $l$ and deal with its injectivity. Namely, we prove that $l$ is injective for isolated quotient singularities over an algebraically 
closed field of characteristic zero (see Proposition 3.11); this is a direct consequence of the fact that $\mathrm{K}_{1}^{s g}(X)=0$ for such singularities. We also note that in our approach the surjectivity of $l$ follows from the topological filtration considerations in subsection 2 and illustrates the interaction between homology and cohomology cycles in dimension zero: skyscraper sheaf of a singular point (homology cycle) is represented by a class of a perfect complex (cohomology cycle).

In general we show that any example where $\mathrm{K}_{0}(X) \rightarrow \mathrm{G}_{0}(X)$ has nonzero kernel will automatically have $\operatorname{Ker}(l) \neq 0$ (Remark 3.12).

4. Homological Bondal-Orlov localization conjecture. Given a variety $X$ with rational singularities and $\pi: Y \rightarrow X$ a resolution of singularities, it is a natural question whether $\pi_{*}$ : $\mathcal{D}^{b}(Y) \rightarrow \mathcal{D}^{b}(X)$ is essentially surjective and whether there is an equivalence $\mathcal{D}^{b}(Y) / \operatorname{Ker}\left(\pi_{*}\right) \simeq$ $\mathcal{D}^{b}(X)$, that is $\mathcal{D}^{b}(X)$ is a Verdier quotient of $\mathcal{D}^{b}(Y)$; this question may be called the BondalOrlov localization conjecture [12]. As a side result, which is morally related, in a certain sense dual to, but not dependent on the singularity category, we prove that Bondal-Orlov localization conjecture holds for quotient singularities (not necessarily isolated) in characteristic zero (Theorem 2.30). This implies in particular that $\pi_{*}: \mathrm{G}_{0}(Y) \rightarrow \mathrm{G}_{0}(X)$ is surjective, which is the "dual" statement to the injectivity $\pi^{*}: \mathrm{K}_{0}(X) \rightarrow \mathrm{K}_{0}(Y)$ for isolated quotient singularities explained in subsection 1 above (but there is no logical link between the two statements).

In the more general setting, to the best of our knowledge it is not known whether the pushforward morphism

$$
\pi_{*}: \mathrm{G}_{0}(Y) \rightarrow \mathrm{G}_{0}(X)
$$

is surjective if $X$ is a variety with rational singularities over an algebraically closed field and $\pi: Y \rightarrow X$ is a resolution. We call this question, as well as the long G-theory exact sequence

$$
\cdots \rightarrow \mathrm{K}_{i}\left(\operatorname{Ker}\left(\pi_{*}\right)\right) \rightarrow \mathrm{G}_{i}(Y) \rightarrow \mathrm{G}_{i}(X) \rightarrow \cdots \rightarrow \mathrm{K}_{0}\left(\operatorname{Ker}\left(\pi_{*}\right)\right) \rightarrow \mathrm{G}_{0}(Y) \rightarrow \mathrm{G}_{0}(X) \rightarrow 0
$$

predicted by the Bondal-Orlov conjecture the Homological Bondal-Orlov conjecture and we hope return to this question in the future.

Acknowledgements. We would like to thank A. Betina, T. Bridgeland, J. Greenlees, A. Efimov, M. Kalck, J. Karmazyn, J. Kass, A. Kuznetsov, N. Pagani, D. Pomerleano, R. Potter, M. Schlichting, P. Sechin, V. Srinivas, P. Stellari, G. Stevenson, B. Totaro, A. Vishik, V. Vologodsky and M. Wemyss for helpful discussions and e-mail communication. E.S. was partially supported by Laboratory of Mirror Symmetry NRU HSE, RF government grant, ag. N 14.641.31.0001.

Notation and conventions. Unless specified otherwise, the schemes we consider are quasiprojective over a field $k$, however most results remain true in the generality of Orlov's (ELF) condition [56]. Furthermore, the base field $k$ is assumed to have characteristic zero; however all general results in Section 1 are true without this assumption. 
If $G$ is a finite group, we write $\widehat{G}$ for the group of characters $\operatorname{Hom}\left(G, k^{*}\right)$. We write $\mathbb{Z}_{n}$ for the cyclic group of order $n$.

All triangulated and dg categories are assumed to be $k$-linear. All functors such as pullback $\pi^{*}$, pushforward $\pi_{*}$ and tensor product $\otimes$ when considered between derived categories are derived functors.

\section{Singularity K-Theory}

1.1. Triangulated and $\mathrm{dg}$ singularity categories. We start by introducing the category whose K-theory we are going to study. Unless stated otherwise, $X$ is a quasi-projective scheme over a field $k$. We write $\mathcal{D}^{b}(X)$ for the bounded derived category of coherent sheaves on $X$ and $\mathcal{D}^{\text {perf }}(X)$ for its subcategory of perfect complexes, which in the quasi-projective case coincides with the subcategory of bounded complexes of locally free sheaves.

Definition 1.1 (Buchweitz [13], Orlov [56]). The triangulated category of singularities of $X$ is the Verdier quotient

$$
\mathcal{D}^{\mathrm{sg}}(X):=\mathcal{D}^{b}(X) / \mathcal{D}^{\text {perf }}(X)
$$

As we will be interested in K-theory of the singularity category, we need to specify a dgenhancement for $\mathcal{D}^{\mathrm{sg}}(X)$ to apply Schlichting's machinery of K-theory of dg-categories.

For that we first recall that $\mathcal{D}^{b}(X)$ has a unique dg-enhancement, up to quasi-equivalence [48, 15]. We denote this dg-enhancement by $\mathcal{D}_{d g}^{b}(X)$. Considering the full dg-subcategory of perfect objects in $\mathcal{D}_{d g}^{b}(X)$, we get a dg-enhancement $\mathcal{D}_{d g}^{\text {perf }}(X)$ of $\mathcal{D}^{\text {perf }}(X)$. Finally, applying the Drinfeld quotient construction [23] to the pair $\mathcal{D}_{d g}^{\text {perf }}(X) \subset \mathcal{D}_{d g}^{b}(X)$ we get a dg-category $\mathcal{D}_{d g}^{\mathrm{sg}}(X)$ which is a dg-enhancement for $\mathcal{D}^{\mathrm{sg}}(X)$.

We note that even though the dg-enhancement of $\mathcal{D}^{\mathrm{sg}}(X)$ may not be unique, our choice is canonical in a way that all enhancements of $\mathcal{D}^{\mathrm{sg}}(X)$ induced by an enhancement of $\mathcal{D}^{b}(X)$ are quasi-equivalent.

Similarly, we consider the singularity category with supports. For any closed $Z \subset X$ let

$$
\mathcal{D}_{Z}^{\mathrm{sg}}(X)=\mathcal{D}_{Z}^{b}(X) / \mathcal{D}_{Z}^{\text {perf }}(X) .
$$

Here $\mathcal{D}_{Z}^{b}(X)$ consists of complexes in $\mathcal{D}^{b}(X)$ acyclic away from $Z$, and $\mathcal{D}_{Z}^{\text {perf }}(X)=\mathcal{D}_{Z}^{b}(X) \cap$ $\mathcal{D}^{\text {perf }}(X)$. A dg-enhancement of $\mathcal{D}^{b}(X)$ induces one for $\mathcal{D}_{Z}^{b}(X)$, and using the Drinfeld quotient construction, $\mathcal{D}_{Z}^{\mathrm{sg}}(X)$ acquires a dg-enhancement $\mathcal{D}_{Z, d g}^{\mathrm{sg}}(X)$.

We now list some properties of the singularity categories, which are due to Orlov. Even though Orlov formulates these results on the triangulated level, they all lift to the dg-enhancements due to the fact that all well-defined derived pull-back and push-forward functors lift to dgenhancements of $\mathcal{D}^{b}(X)$ and $\mathcal{D}^{\text {perf }}(X)[63$. 
Proposition 1.2 (Orlov [56]). Let $j: U \subset X$ be an open embedding such that $\operatorname{Sing}(X) \subset U$. Then

$$
j^{*}: \mathcal{D}^{\mathrm{sg}}(X) \stackrel{\sim}{\rightarrow} \mathcal{D}^{\mathrm{sg}}(U)
$$

is an equivalence, induced by a functor between dg-enhancements.

Theorem 1.3 (Knörrer periodicity, Orlov [56]). Let $X / k$ be a smooth quasi-projective scheme and let $f: X \rightarrow \mathbb{A}^{1}$ be a non-zero morphism. Define $g=f+x y: X \times \mathbb{A}^{2} \rightarrow \mathbb{A}^{1}$. Let $Z_{f}=f^{-1}(\{0\})$ and $Z_{g}=g^{-1}(\{0\})$, and let $W=Z_{f} \times\{0\} \times \mathbb{A}^{1} \subset X \times \mathbb{A}^{2}$. Furthermore, denote by $i: W \hookrightarrow Z_{g}$ the inclusion and $p: W \rightarrow Z_{f}$ the flat projection. Then

$$
i_{*} p^{*}: \mathcal{D}^{\mathrm{sg}}\left(Z_{f}\right) \rightarrow \mathcal{D}^{\mathrm{sg}}\left(Z_{g}\right)
$$

is an equivalence of triangulated categories induced by a functor between dg-enhancements.

Recall that a full triangulated subcategory $\mathcal{T}$ of a triangulated category $\mathcal{D}$ is called dense if any object in $\mathcal{D}$ is a direct summand of an object in $\mathcal{T}$.

Proposition 1.4 (Orlov [57]). For any closed subset $Z \subseteq X$, the induced functor

$$
\mathcal{D}_{Z}^{\mathrm{sg}}(X) \rightarrow \mathcal{D}^{\mathrm{sg}}(X)
$$

is fully faithful and is induced by a functor between dg-enhancements. Furthermore, if $\operatorname{Sing}(X) \subset$ $Z$, then this functor has a dense image.

If $\mathcal{D}$ is a triangulated category, then we write $\overline{\mathcal{D}}$ for the idempotent completion of $\mathcal{D}$; $\overline{\mathcal{D}}$ is a triangulated category by [4]. If $\mathcal{D}$ admits a dg-enhancement, then the fully faithful functor $\mathcal{D} \subset \overline{\mathcal{D}}$ is induced by a dg-functor (see e.g. [6, 1.6.2]).

Theorem 1.5 (Orlov [57]). Assume that the formal completions of $X$ and $X^{\prime}$ along $\operatorname{Sing}(X)$ and $\operatorname{Sing}\left(X^{\prime}\right)$ respectively are isomorphic. Then we have equivalences

$$
\mathcal{D}_{\operatorname{Sing}(X)}^{\mathrm{sg}}(X) \simeq \mathcal{D}_{\operatorname{Sing}\left(X^{\prime}\right)}^{\mathrm{sg}}\left(X^{\prime}\right)
$$

and

$$
\overline{\mathcal{D}^{\operatorname{sg}}(X)} \simeq \overline{\mathcal{D}^{\operatorname{sg}}\left(X^{\prime}\right)}
$$

induced by functors between dg-enhancements.

In light of our interest in idempotent completions we will also need the following celebrated result by Thomason.

Theorem 1.6 (Thomason, Theorem 2.1 in [72]). Let $\mathcal{D}$ be an essentially small triangulated category, then there is a one-to-one correspondence

$\{\mathcal{T} \subseteq \mathcal{D} \mid \mathcal{T}$ dense strictly full triang. subcat. $\} \stackrel{1: 1}{\longleftrightarrow}\left\{H \subseteq \mathrm{K}_{0}(\mathcal{D}) \mid H\right.$ subgroup $\}$. 
The correspondence sends strictly full dense subcategories $\mathcal{T} \subseteq \mathcal{D}$ to the image of $\mathrm{K}_{0}(\mathcal{T})$ in $\mathrm{K}_{0}(\mathcal{D})$ and the inverse sends a subgroup $H$ of $\mathrm{K}_{0}(\mathcal{D})$ to the full triangulated subcategory $\mathcal{D}_{H}$, where $\mathcal{D}_{H}:=\left\{A \in \mathcal{D} \mid[A] \in H \subseteq \mathrm{K}_{0}(\mathcal{D})\right\}$.

1.2. K-Theory of the singularity category. Schlichting's construction of the K-theory spectrum [61, 62] can be applied to produce K-theory groups $\mathbb{K}_{i}(\mathcal{C}), i \in \mathbb{Z}$ for a $k$-linear pretriangulated dg-category $\mathcal{C}$.

The $\mathbb{K}_{i}$ groups are covariantly functorial for dg-functors; we summarize their properties as follows. For a pretriangulated dg-category $\mathcal{C}$ we write $H^{0}(\mathcal{C})$ for its triangulated homotopy category.

(0) $\mathbb{K}_{0}(\mathcal{C})$ is the Grothendieck group of the idempotent completion of $H^{0}(\mathcal{C})$.

(1) If $\mathcal{C} \rightarrow \mathcal{C}^{\prime}$ induces a fully faithful embedding $H^{0}(\mathcal{C}) \rightarrow H^{0}\left(\mathcal{C}^{\prime}\right)$ with a dense image, then all $\mathbb{K}_{i}(\mathcal{C}) \rightarrow \mathbb{K}_{i}\left(\mathcal{C}^{\prime}\right)$ are isomorphisms.

(2) If $\mathcal{A} \rightarrow \mathcal{B} \rightarrow \mathcal{C}$ induces a fully faithful embedding $H^{0}(\mathcal{A}) \rightarrow H^{0}(\mathcal{B})$ such that $H^{0}(\mathcal{A})$ is the kernel of $H^{0}(\mathcal{B}) \rightarrow H^{0}(\mathcal{C})$ and a fully faithful functor $H^{0}(\mathcal{B}) / H^{0}(\mathcal{A}) \rightarrow H^{0}(\mathcal{C})$ with a dense image, then there is a long exact sequence

$$
\cdots \rightarrow \mathbb{K}_{i}(\mathcal{A}) \rightarrow \mathbb{K}_{i}(\mathcal{B}) \rightarrow \mathbb{K}_{i}(\mathcal{C}) \rightarrow \mathbb{K}_{i-1}(\mathcal{A}) \rightarrow \ldots
$$

(3) $\mathbb{K}_{i}\left(\mathcal{D}_{d g}^{b}(X)\right)$ are isomorphic to $\mathrm{G}_{i}(X)$, Quillen's G-theory, that is K-theory of coherent sheaves. In particular, $\mathbb{K}_{i}\left(\mathcal{D}^{b}(X)\right)=0$ for $i<0$.

(4) $\mathbb{K}_{i}\left(\mathcal{D}_{d g}^{\text {perf }}(X)\right)$ are isomorphic to $\mathrm{K}_{i}(X)$, the Thomason-Trobaugh K-theory [73], which under our assumptions on $X$ (quasi-projective scheme over a field) are isomorphic to Quillen's K-theory of vector bundles.

Remark 1.7. It is well-known that K-theory of triangulated categories satisfying the axioms analogous to those listed above cannot be defined [60]; the counterexample is provided by the two singularity categories of schemes which are equivalent as triangulated categories but are forced to have non-isomorphic higher $\mathrm{K}$-groups if one assumes the long exact $\mathrm{K}$-theory sequence for Verdier quotients of triangulated categories.

Definition 1.8. We define the singularity K-theory groups of $X$ by

$$
\mathrm{K}_{i}^{s g}(X)= \begin{cases}\mathbb{K}_{i}\left(\mathcal{D}_{d g}^{\mathrm{sg}}(X)\right), & i \neq 0 \\ \mathrm{~K}_{0}\left(\mathcal{D}_{d g}^{\mathrm{sg}}(X)\right), & i=0\end{cases}
$$

and we also define $\mathbb{K}_{0}^{s g}(X)=\mathbb{K}_{0}\left(\mathcal{D}^{\mathrm{sg}}(X)\right)$.

Remark 1.9. We make a special consideration for $i=0$ since by property (0) above $\mathbb{K}_{0}^{s g}(X)$ is in fact the Grothendieck group of the idempotent completion of $\overline{\mathcal{D}^{\mathrm{sg}}(X)}$, and not of $\mathcal{D}^{\mathrm{sg}}(X)$ itself. By Theorem [1.6 we have $\mathrm{K}_{0}^{s g}(X) \subset \mathbb{K}_{0}^{s g}(X)$. On the other hand by property (1) it is true that for $i \neq 0, \mathrm{~K}_{i}^{s g}(X)=\mathbb{K}_{i}\left(\mathcal{D}_{d g}^{\mathrm{sg}}(X)\right) \simeq \mathbb{K}_{i}\left(\overline{\mathcal{D}_{d g}^{\mathrm{sg}}(X)}\right)$. 
Let us write $\mathrm{PD}: \mathrm{K}_{i}(X) \rightarrow \mathrm{G}_{i}(X)$ for the canonical "Poincaré duality" morphism induced by $\mathcal{D}_{d g}^{\text {perf }}(X) \subset \mathcal{D}_{d g}^{b}(X)$. Our main motivation in defining the singularity K-theory is for studying this map.

Lemma 1.10 (Singularity K-theory exact sequences). We have exact sequences

$$
\begin{gathered}
\cdots \rightarrow \mathrm{K}_{i}(X) \stackrel{\mathrm{PD}}{\rightarrow} \mathrm{G}_{i}(X) \rightarrow \mathrm{K}_{i}^{s g}(X) \rightarrow \cdots \rightarrow \mathrm{K}_{0}(X) \stackrel{\text { PD }}{\rightarrow} \mathrm{G}_{0}(X) \rightarrow \mathrm{K}_{0}^{s g}(X) \rightarrow 0, \\
0 \rightarrow \mathrm{K}_{0}^{s g}(X) \rightarrow \mathbb{K}_{0}^{s g}(X) \rightarrow \mathrm{K}_{-1}(X) \rightarrow 0 .
\end{gathered}
$$

and isomorphisms for $j \geq 1$

$$
\mathrm{K}_{-j}^{s g}(X) \simeq \mathrm{K}_{-j-1}(X)
$$

Proof. The statement follows from a single K-theory sequence using the properties of Schlichting $\mathrm{K}$-groups given above and the fact that the image of $\mathrm{G}_{0}(X)$ in $\mathbb{K}_{0}^{s g}(X)$ is $\mathrm{K}_{0}^{s g}(X)$.

We record the following well-known result:

Lemma 1.11. $\mathcal{D}^{\mathrm{sg}}(X)$ is idempotent complete if and only if $\mathrm{K}_{-1}(X)=0$.

Proof. Using (1.2) we see that vanishing of $\mathrm{K}_{-1}(X)$ is equivalent to $\mathrm{K}_{0}\left(\mathcal{D}^{\mathrm{sg}}(X)\right)=\mathrm{K}_{0}\left(\overline{\mathcal{D}^{\operatorname{sg}}(X)}\right)$ which implies $\mathcal{D}^{\mathrm{sg}}(X)=\overline{\mathcal{D}^{\mathrm{sg}}(X)}$ by the theorem of Thomason (Theorem 1.61).

Similarly to the definition of the singularity K-theory, for every closed $Z \subset X$ we consider the singularity K-theory with supports defined by

$$
\mathrm{K}_{i}^{s g}(X \text { on } Z)= \begin{cases}\mathbb{K}_{i}\left(\mathcal{D}_{Z, d g}^{\mathrm{sg}}(X)\right), & i \neq 0 \\ \mathrm{~K}_{0}\left(\mathcal{D}_{Z, d g}^{\mathrm{sg}}(X)\right), & i=0\end{cases}
$$

Lemma 1.12. If $\operatorname{Sing}(X) \subset Z$, then we have natural isomorphisms $\mathrm{K}_{i}^{s g}(X$ on $Z) \simeq \mathrm{K}_{i}^{s g}(X)$ for $i \neq 0$ and $\mathrm{K}_{0}^{s g}(X$ on $Z) \rightarrow \mathrm{K}_{0}^{s g}(X)$ is injective.

Proof. Follows from Proposition 1.4 and property (1) of Schlichting's K-theory.

Remark 1.13. There are exact sequences for singularity $\mathrm{K}$-theory with supports analogous to (1.1), (1.2); note that $\mathrm{K}_{i}\left(\mathcal{D}_{d g, Z}^{\text {perf }}(X)\right)=\mathrm{K}_{i}(X$ on $Z)$ are the Thomason-Trobaugh $\mathrm{K}$-theory groups [73] while $\mathrm{K}_{i}\left(\mathcal{D}_{d g, Z}^{b}(X)\right) \simeq \mathrm{G}_{i}(Z)$ is Quillen's $\mathrm{G}$-theory [58].

We now discuss functoriality properties of $\mathrm{K}_{i}^{s g}$.

Lemma 1.14. $\mathrm{K}_{i}^{\text {sg }}$ are contravariantly functorial for morphisms of finite Tor-dimension, and are covariantly functorial for proper morphisms of finite Tor-dimension.

Proof. This holds because of the triangulated singularity categories have this functoriality [56], and since the pull-back and pushforward functors are induced by dg-enhancements [63]. 
Lemma 1.15. Let $U$ be an open subscheme of $X$ containing the singular locus $\operatorname{Sing}(X)$. Then the inclusion $j: U \rightarrow X$ induces an isomorphism

$$
j^{*}: \mathrm{K}_{i}^{s g}(X) \simeq \mathrm{K}_{i}^{s g}(U)
$$

for all $i \in \mathbb{Z}$.

Proof. By Proposition $1.2 j^{*}$ is a quasi-equivalence on $\mathrm{dg}$ singularity categories, so it must induce an isomorphism on K-theory groups.

Lemma 1.16. Let $X$ be a quasi-projective variety, and let $p: V \rightarrow X$ be a vector bundle over $X$. Then we have an isomorphism

$$
p^{*}: \mathrm{K}_{0}^{s g}(X) \simeq \mathrm{K}_{0}^{s g}(V) .
$$

Proof. Let $i: X \rightarrow V$ the zero section. Since $p$ is flat and $i$ is a regular embedding, both morphisms $p$ and $i$ are of finite Tor dimension, so by Lemma 1.14 we have pull-back homomorphisms $p^{*}: \mathrm{K}_{i}^{s g}(X) \rightarrow \mathrm{K}_{i}^{s g}(V)$ and $i^{*}: \mathrm{K}_{i}^{s g}(V) \rightarrow \mathrm{K}_{i}^{s g}(X)$, and $i^{*}$ is left-inverse to $p^{*}$, in particular $p^{*}$ is injective.

On the other hand, from the diagram

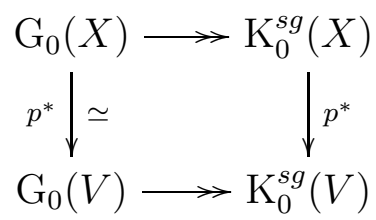

we see immediately that $p^{*}: \mathrm{K}_{0}^{s g}(X) \rightarrow \mathrm{K}_{0}^{s g}(V)$ is surjective as well.

Remark 1.17. The functors $\mathrm{K}_{i}^{s g}$ are not homotopy invariant for $i \neq 0$ in general. Consider for example the case of $\mathrm{K}_{1}^{s g}$; if we have $\mathrm{K}_{1}^{s g}\left(X \times \mathbb{A}^{1}\right) \simeq \mathrm{K}_{1}^{s g}(X)$, then using the five-lemma applied to the five bottom terms of the sequence (1.1), we would deduce that $\mathrm{K}_{0}\left(X \times \mathbb{A}^{1}\right) \simeq \mathrm{K}_{0}(X)$ which typically does not hold for singular varieties.

We will now present a method to compute $\mathrm{K}_{j}^{s g}(X)$ for a special class of schemes which we call $\mathbb{A}^{1}$-contractible. This approach generalizes the so-called Swan-Weibel homotopy trick, which is used to show that normal graded domains have vanishing Picard group [51, Lemma 5.1].

Definition 1.18. We say that $X$ is $\mathbb{A}^{1}$-contractible, if there exists a morphism $H: X \times \mathbb{A}^{1} \rightarrow X$ such that $\left.H\right|_{X \times 1}$ is the identity map and $\left.H\right|_{X \times 0}$ is a constant rational point $x_{0} \in X$. We also say that $H$ is a contraction of $X$.

Lemma 1.19. The following affine schemes are $\mathbb{A}^{1}$-contractible:

(1) $\mathbb{A}^{n} / G$, where $G$ acts linearly on $\mathbb{A}^{n}$

(2) $V(f) \subset \mathbb{A}^{n}$, where $f \in k\left[x_{1}, \ldots, x_{n}\right]$ is a weighted homogeneous polynomial 
Proof. (1) $\mathbb{A}^{n}$ admits a $G$-equivariant contraction $H_{\mathbb{A}^{n}}: \mathbb{A}^{n} \times \mathbb{A}^{1} \rightarrow \mathbb{A}^{n}, H(v, t)=t v(G$ acts trivially on the $\mathbb{A}^{1}$ factor), which induces a contraction $H: \mathbb{A}^{n} / G \times \mathbb{A}^{1} \rightarrow \mathbb{A}^{n} / G$.

(2) By assumption the algebra $k[V(f)]=k\left[x_{1}, \ldots, x_{n}\right] /(f)$ is positively graded. Let $w_{i}>0$ be the weight of $x_{i}$. Then the $k$-algebra morphism

$$
k[X] \rightarrow k[X, t], \quad x_{i} \mapsto t^{w_{i}} \cdot x_{i}
$$

is well-defined and induces a contraction for $V(f)$.

Proposition 1.20. Let $X$ be $\mathbb{A}^{1}$-contractible.

(1) For every $j \geq 0$ the canonical map $\mathrm{PD}: \mathrm{K}_{j}(X) \rightarrow \mathrm{G}_{j}(X)$ factors as a composition $\mathrm{K}_{j}(X) \stackrel{x_{0}^{*}}{\rightarrow} \mathrm{K}_{j}(\operatorname{Spec}(k)) \stackrel{p^{*}}{\rightarrow} \mathrm{G}_{j}(X)$, where $p: X \rightarrow \operatorname{Spec}(k)$ is the structure morphism.

(2) There is a natural isomorphism

$$
\mathrm{K}_{0}^{s g}(X) \simeq \mathrm{G}_{0}(X) /\left(\mathbb{Z} \cdot\left[\mathcal{O}_{X}\right]\right)
$$

(3) If $X$ has a smooth rational point $x_{1} \in X$, then

$$
\mathrm{G}_{0}(X)=\mathbb{Z} \cdot\left[\mathcal{O}_{X}\right] \oplus \mathrm{K}_{0}^{s g}(X)
$$

and for every $j \geq 1$ there is a short exact sequence

$$
0 \rightarrow \operatorname{Coker}\left(\mathrm{K}_{j}(k) \stackrel{p^{*}}{\rightarrow} \mathrm{G}_{j}(X)\right) \rightarrow \mathrm{K}_{j}^{s g}(X) \rightarrow \operatorname{Ker}\left(\mathrm{K}_{j-1}(X) \stackrel{x_{0}^{*}}{\rightarrow} \mathrm{K}_{j-1}(k)\right) \rightarrow 0 .
$$

Proof. (1) The proof relies on the fact that the canonical map PD commutes with pull-backs of finite Tor dimension as well as on homotopy invariance of G-theory. Let us write $i_{0}, i_{1}$ for the two embeddings of $X$ into $X \times \mathbb{A}^{1}$ corresponding to $0,1 \in \mathbb{A}^{1}$. These embeddings define Cartier divisors, in particular are regular, hence of finite Tor-dimension.

In the computation below we use the notation $K(f)$ and $G(f)$ for the pull-backs on $\mathrm{K}$ and G-theory respectively, and $\mathrm{PD}_{Z}$ for the canonical Poincaré duality map $\mathrm{K}_{0}(Z) \rightarrow \mathrm{G}_{0}(Z)$ on any $Z$. We compute:

$$
\begin{aligned}
\mathrm{PD}_{X} & =\mathrm{PD}_{X} \circ K\left(i_{1}\right) \circ K(H) \\
& =G\left(i_{1}\right) \circ \mathrm{PD}_{X \times \mathbb{A}^{1}} \circ K(H) \\
& =G\left(i_{0}\right) \circ \mathrm{PD}_{X \times \mathbb{A}^{1}} \circ K(H) \\
& =\mathrm{PD}_{X} \circ K\left(i_{0}\right) \circ K(H) \\
& =\mathrm{PD}_{X} \circ K(p) \circ K\left(x_{0}\right) \\
& =G(p) \circ \mathrm{PD}_{\mathrm{Spec}(k)} \circ K\left(x_{0}\right)
\end{aligned}
$$

which is what we had to establish.

Let us now compute $\operatorname{Ker}(\mathrm{PD})$, Coker(PD) for $\mathrm{PD}: \mathrm{K}_{j}(X) \rightarrow \mathrm{G}_{j}(X)$ using (1). The map $x_{0}^{*}$ is always surjective ( since $\left.x_{0}^{*} \circ p^{*}=\mathrm{id}_{\mathrm{K}_{j}(k)}\right)$, hence $\operatorname{Coker}(\mathrm{PD})=\operatorname{Coker}\left(p^{*}\right)$, and applying this to $j=0$ using the K-theory short exact sequence (1.1) we get (2). 
If in addition $X$ admits a smooth rational point $x_{1}$, then the map $p^{*}$ is injective (since $x_{1}^{*} \circ p^{*}=\mathrm{id}_{\mathrm{K}_{j}(k)}$ for the pull-back $x_{1}^{*}: \mathrm{G}_{j}(X) \rightarrow \mathrm{K}_{j}(k)$ for the regular embedding of $x_{1}$ into $\left.X\right)$, hence (1) implies $\operatorname{Ker}(\mathrm{PD})=\operatorname{Ker}\left(x_{0}^{*}\right)$.

Once we have identified the kernel and cokernel of PD, (3) follows from the K-theory long exact sequence (1.1).

In the two examples below we consider $\mathbb{A}^{1}$-contractible schemes with no smooth rational points.

Example 1.21. $X=\operatorname{Spec}\left(k[\epsilon] / \epsilon^{n}\right)$ is $\mathbb{A}^{1}$-contractible by Lemma 1.19 (2). In this case the canonical map $\mathrm{K}_{0}(X) \rightarrow \mathrm{G}_{0}(X)$ is $\mathbb{Z} \stackrel{\cdot n}{\rightarrow} \mathbb{Z}$ and $\mathrm{K}_{0}^{s g}(X)=\mathbb{Z}_{n}$.

Example 1.22. Let $X$ be an affine curve $x^{2}+y^{2}=0$ over $k=\mathbb{R}$, the real numbers.

To compute $\mathrm{G}_{0}(X)$ we consider a compactification $X \subset \bar{X}$, where $\bar{X}$ is given by equation $X^{2}+Y^{2}=0$ in the real projective plane $\mathbb{P}_{\mathbb{R}}^{2}$ with coordinates $X, Y, Z$. The complement $\bar{X} \backslash X$ is the single closed (non-rational) point at infinity $\infty \in \bar{X}$; as a subscheme $\infty$ is isomorphic to $\operatorname{Spec}(\mathbb{C})$.

It is easy to see that there is an isomorphism

$$
\mathrm{G}_{0}(\bar{X}) \simeq \mathbb{Z} \oplus \mathbb{Z}, \quad[\mathcal{F}] \mapsto(\operatorname{rk}(\mathcal{F}), \operatorname{deg}(\mathcal{F}))
$$

(surjectivity is obvious, while injectivity boils down to the fact that every class of a skyscraper sheaf of a closed point $x \in \bar{X}$ is a multiple of the class of the skyscraper sheaf of the rational point $[0: 0: 1]$, and this can be checked using the fact that $\left.\mathrm{CH}_{0}(\bar{X})=\mathbb{Z}\right)$.

We write the $\mathrm{G}$-theory localization sequence for $X \subset \bar{X}$ :

$$
\mathrm{G}_{0}(\operatorname{Spec}(\mathbb{C})) \rightarrow \mathrm{G}_{0}(\bar{X}) \rightarrow \mathrm{G}_{0}(X) \rightarrow 0 .
$$

Under the isomorphism $\mathrm{G}_{0}(\bar{X})=\mathbb{Z} \oplus \mathbb{Z}$ the class of structure sheaf of the point at infinity corresponds to $(0,2)$; we deduce that $\mathrm{G}_{0}(X)=\mathbb{Z} \oplus \mathbb{Z}_{2}$ given by the rank map and degree modulo two.

The curve $X$ is $\mathbb{A}^{1}$-contractible by Lemma 1.19 (2) so that by Proposition 1.20 (2) we obtain

$$
\mathrm{K}_{0}^{s g}(X)=\mathrm{G}_{0}(X) / \mathbb{Z} \cdot\left[\mathcal{O}_{X}\right]=\mathbb{Z}_{2},
$$

generated by the class of the structure sheaf of the rational point $(0,0) \in X$.

1.3. Topological filtration on $\mathrm{K}_{0}^{s g}$. We introduce and study the topological filtration on $\mathrm{K}_{0}^{s g}(X)$, that is the filtration given by the codimension of support. Note that unlike the case of objects from $\mathcal{D}^{b}(X)$, the support of an object in $\mathcal{D}^{\mathrm{sg}}(X)$ is NOT well-defined, as the following example demonstrates.

Example 1.23. Let $X=\{x y=0\} \subset \mathbb{A}^{2}$ be the union of two $\mathbb{A}^{1}$-lines over $k$. Denote the two affine lines by $L_{1}=\mathbb{A}^{1} \times 0 \subset X$ and $L_{2}=0 \times \mathbb{A}^{1} \subset X$. The structure sheaves of $L_{1}$ and $L_{2}$ 
correspond to quotient rings $k[x]=k[x, y] /(y)$ and $k[y]=k[x, y] /(x)$ respectively. We have an exact sequence of $k[x, y] /(x y)$-modules

$$
0 \rightarrow k[x] \stackrel{x}{\rightarrow} k[x, y] /(x, y) \rightarrow k[y] \rightarrow 0
$$

which translates into a distinguished triangle in $\mathcal{D}^{b}(X)$

$$
\mathcal{O}_{L_{1}} \rightarrow \mathcal{O}_{X} \rightarrow \mathcal{O}_{L_{2}} \rightarrow \mathcal{O}_{L_{1}}[1]
$$

and yields an isomorphism $\mathcal{O}_{L_{2}} \simeq \mathcal{O}_{L_{1}}$ [1] of objects $\mathcal{D}^{\mathrm{sg}}(X)$ (the shift [1] is two-periodic in this example); sheaf-theoretic supports of these two objects are different.

We can speak about codimension of support of an object of $\mathcal{D}^{\text {sg }}(X)$ without defining the support itself. Let $X$ be a quasi-projective scheme with all irreducible components of the same dimension $n$.

Recall that $\mathrm{K}_{0}(X), \mathrm{G}_{0}(X)$ admit the so-called topological filtration (also called the coniveau or the codimension filtration), which goes back to Grothendieck and is defined as follows. The class $\alpha \in \mathrm{G}_{0}(X)$ (resp. $\mathrm{K}_{0}(X)$ ) belongs to $F^{i} \mathrm{G}_{0}(X)$ (resp. $F^{i} \mathrm{~K}_{0}(X)$ ) if $\alpha$ can be represented by a bounded complex of coherent sheaves (resp. locally free sheaves) whose support has codimension at least $i$. It is clear from the definitions that the canonical map PD : $\mathrm{K}_{0}(X) \rightarrow$ $\mathrm{G}_{0}(X)$ maps $F^{i} \mathrm{~K}_{0}(X)$ to $F^{i} \mathrm{G}_{0}(X)$.

We consider the natural quotient homomorphism $Q: \mathrm{G}_{0}(X) \rightarrow \mathrm{K}_{0}^{s g}(X)$ and define

$$
F^{i} \mathrm{~K}_{0}^{s g}(X)=Q\left(F^{i} \mathrm{G}_{0}(X)\right) .
$$

This gives a filtration

$$
0=F^{n+1} \mathrm{~K}_{0}^{s g}(X) \subseteq F^{n} \mathrm{~K}_{0}^{s g}(X) \subseteq \ldots \subseteq F^{1} \mathrm{~K}_{0}^{s g}(X) \subseteq F^{0} \mathrm{~K}_{0}^{s g}(X)=\mathrm{K}_{0}^{s g}(X) .
$$

Explicitly, we say that a class $\alpha \in \mathrm{K}_{0}^{s g}(X)$ has codimension at least $i$ or that $\alpha \in F^{i} \mathrm{~K}_{0}^{s g}(X)$, if $\alpha$ can be represented by a complex of coherent sheaves $\mathcal{E}$ on $X$ whose cohomology sheaves are supported in codimension $i$.

It follows from definitions that we have canonical isomorphisms

$$
F^{i} \mathrm{~K}_{0}^{s g}(X) \simeq \frac{F^{i} \mathrm{G}_{0}(X)}{F^{i} \mathrm{G}_{0}(X) \cap \operatorname{PD}\left(\mathrm{K}_{0}(X)\right)}
$$

We let $\operatorname{gr}^{i} \mathrm{~K}_{0}^{s g}(X)=F^{i} \mathrm{~K}_{0}^{s g}(X) / F^{i+1} \mathrm{~K}_{0}^{s g}(X)$, and similarly for $\mathrm{K}_{0}(X), \mathrm{G}_{0}(X)$. We have a natural surjection

$$
\operatorname{gr}^{i} \mathrm{G}_{0}(X) \rightarrow \operatorname{gr}^{i} \mathrm{~K}_{0}^{s g}(X)
$$

and canonical isomorphisms

$$
\operatorname{gr}^{i} \mathrm{~K}_{0}^{s g}(X) \simeq \frac{F^{i} \mathrm{G}_{0}(X)}{F^{i+1} \mathrm{G}_{0}(X)+\left(F^{i} \mathrm{G}_{0}(X) \cap \operatorname{PD}\left(\mathrm{K}_{0}(X)\right)\right)} .
$$


Proposition 1.24. Let $X / k$ be a connected reduced quasi-projective scheme with all irreducible components of the same dimension $n$.

(1) Let $N$ be the number of irreducible components of $X$. Then $\operatorname{gr}^{0} \mathrm{~K}_{0}^{s g}(X)=\mathbb{Z}^{N-1}$. In particular $\operatorname{gr}^{0} \mathrm{~K}_{0}^{s g}(X)=0$ if and only if $X$ is irreducible.

(2) If $X$ is irreducible and normal then $\operatorname{gr}^{1} \mathrm{~K}_{0}^{s g}(X) \simeq \mathrm{Cl}(X) / \operatorname{Pic}(X)$. In particular in this case $\operatorname{gr}^{1} \mathrm{~K}_{0}^{s g}(X)=0$ if and only if $X$ is factorial.

(3) For any $i \geq 0$ there is a natural surjective homomorphism $\mathrm{CH}_{n-i}(X) \rightarrow \operatorname{gr}^{i} \mathrm{~K}_{0}^{s g}(X)$.

(4) If $k$ is algebraically closed, then $\operatorname{gr}^{n} \mathrm{~K}_{0}^{s g}(X)=0$.

Proof. (1) Using $i=0$ case of (1.6) we obtain

$$
\operatorname{gr}^{0} \mathrm{~K}_{0}^{s g}(X) \simeq \frac{\mathrm{G}_{0}(X)}{F^{1} \mathrm{G}_{0}(X)+\operatorname{PD}\left(\mathrm{K}_{0}(X)\right)} .
$$

We have $\operatorname{gr}^{0} \mathrm{G}_{0}(X)=\mathbb{Z}^{N}$, where the isomorphism is given by generic rank at the irreducible components. Since $X$ is connected, a locally-free sheaf has the same rank at each point, and the image of the composition $\mathrm{K}_{0}(X) \stackrel{\mathrm{PD}}{\rightarrow} \mathrm{G}_{0}(X) \rightarrow \mathrm{gr}^{0} \mathrm{G}_{0}(X)$ consists of $\mathbb{Z}$ embedded into $\mathbb{Z}^{N}$ diagonally, since $X$ is reduced. We conclude that $\operatorname{gr}^{0} \mathrm{~K}_{0}^{s g}(X)=\mathbb{Z}^{N-1}$.

(2) Since $X$ is irreducible, we have canonical splittings $\mathrm{K}_{0}(X)=\mathbb{Z} \oplus F^{1} \mathrm{~K}_{0}(X)$ and $\mathrm{G}_{0}(X)=$ $\mathbb{Z} \oplus F^{1} \mathrm{G}_{0}(X)$, which are respected by $\mathrm{PD}$ so that from (1.6) we deduce

$$
\operatorname{gr}^{1} \mathrm{~K}_{0}^{s g}(X) \simeq \frac{F^{1} \mathrm{G}_{0}(X)}{F^{2} \mathrm{G}_{0}(X)+\left(F^{1} \mathrm{G}_{0}(X) \cap \operatorname{PD}\left(\mathrm{K}_{0}(X)\right)\right)} \simeq \frac{F^{1} \mathrm{G}_{0}(X)}{F^{2} \mathrm{G}_{0}(X)+\operatorname{PD}\left(F^{1} \mathrm{~K}_{0}(X)\right)} .
$$

By [32, Remark 1 on Page 126], we have a natural isomorphism $\operatorname{gr}^{1} \mathrm{~K}_{0}(X)=\operatorname{Pic}(X)$. Since $X$ is normal so that its singular locus has codimension at least two we also get the following isomorphisms

$$
\operatorname{gr}^{1} \mathrm{G}_{0}(X)=\operatorname{gr}^{1} \mathrm{G}_{0}(X \backslash \operatorname{Sing}(X))=\operatorname{Pic}(X \backslash \operatorname{Sing}(X))=\operatorname{Cl}(X) .
$$

It follows that the image of $F^{1} \mathrm{~K}_{0}(X)$ in $\operatorname{gr}^{1} \mathrm{G}_{0}(X)=\mathrm{Cl}(X)$ is equal to $\operatorname{Pic}(X)$, and we get $\operatorname{gr}^{1} \mathrm{~K}_{0}^{s g}(X)=\mathrm{Cl}(X) / \operatorname{Pic}(X)$.

Finally, $X$ is factorial if and only if $\operatorname{Pic}(X)=\mathrm{Cl}(X)$ which is equivalent to $\operatorname{gr}^{1} \mathrm{~K}_{0}^{s g}(X)=0$.

(3) There is a surjection $\mathrm{CH}_{n-i}(X) \rightarrow \operatorname{gr}^{i} \mathrm{G}_{0}(X)$, sending the class of an $(n-i)$-dimensional subvariety to the structure sheaf of this variety (see SGA X [11] or [33, Lemma 3.8, Theorem 3.9]), and composing it with the surjection $\operatorname{gr}^{i} \mathrm{G}_{0}(X) \rightarrow \operatorname{gr}^{i} \mathrm{~K}_{0}^{s g}(X)$ gives the desired homomorphism.

(4) This is a simple Moving Lemma argument. Assume first that $X$ is irreducible. We fix a closed subvariety $Z \subsetneq X$ containing the singular locus. By De Jong's work (see [22], Theorem 4.1) there is a proper surjective and generically finite morphism $\pi: Y \rightarrow X$ where $Y$ is a smooth irreducible and quasi-projective variety. Let $E=\pi^{-1}(Z) \subset Y$.

Let us show that for every closed point $x \in X$ there is a point $x^{\prime} \in X \backslash Z$ such that $\left[\mathcal{O}_{x}\right]=\left[\mathcal{O}_{x^{\prime}}\right] \in \mathrm{G}_{0}(X)$. Indeed, since we assume that $k$ is algebraically closed, there is a closed 
point $y \in Y$ such that $\pi(y)=x$, and using a simple argument (e.g. reducing to the case when $Y$ is a curve, or using the Moving Lemma [16] for Chow groups), we can find $y^{\prime} \in Y \backslash E$ such that $\left[\mathcal{O}_{y}\right]=\left[\mathcal{O}_{y^{\prime}}\right] \in \mathrm{G}_{0}(Y)$. Pushing forward this equality to $X$ we get $\left[\mathcal{O}_{x}\right]=\left[\mathcal{O}_{x^{\prime}}\right] \in \mathrm{G}_{0}(X)$, where $x^{\prime}=\pi\left(y^{\prime}\right)$. Since the structure sheaves of non-singular points are perfect complexes, we get $\left[\mathcal{O}_{x}\right]=\left[\mathcal{O}_{x^{\prime}}\right]=0 \in \mathrm{K}_{0}^{s g}(X)$. Finally, every class of a zero-dimensional complex $[\mathcal{F}] \in \mathrm{G}_{0}(X)$ is a linear combination of structure sheaves of closed points, and this shows that $F^{n} \mathrm{~K}_{0}^{s g}(X)=0$ if $X$ is irreducible.

If $X$ is not irreducible, the result is obtained by applying the argument above to each of the irreducible components of $X_{i} \subset X$ with respect to $Z=X_{i} \cap \operatorname{Sing}(X)$.

Corollary 1.25. If $k$ is algebraically closed, $x \in X$ is a closed point and $\mathcal{O}_{x}$ its structure sheaf, then the image of $\left[\mathcal{O}_{x}\right] \in \mathrm{G}_{0}(X)$ in $\mathrm{K}_{0}^{s g}(X)$ is zero.

Proof. This is equivalent to Proposition 1.24 (4).

Remark 1.26. The result of the Corollary does not hold if $k$ is not algebraically closed: see Example 1.22, where $X / \mathbb{R}$ is a curve, $\mathrm{K}_{0}^{s g}(X)=\mathbb{Z}_{2}$ and the generator is supported in codimension one.

Corollary 1.27. If $k$ is an algebraically closed field, and $X$ has isolated singularities then $\mathrm{K}_{0}^{s g}(X$ on $\operatorname{Sing}(X))=0$.

Proof. By definition $\mathrm{K}_{0}^{s g}(X$ on $\operatorname{Sing}(X))=\mathrm{K}_{0}\left(\mathcal{D}_{\operatorname{Sing}(X)}^{\mathrm{sg}}(X)\right)$, and from Proposition 1.4 and Theorem 1.6 it follows that

$$
\mathrm{K}_{0}^{s g}(X \text { on } \operatorname{Sing}(X)) \subset \mathrm{K}_{0}^{s g}(X)
$$

is generated by classes of coherent sheaves supported on the singular locus, so the first group has to be zero by Proposition 1.24 (4) as the singular locus is zero-dimensional by assumption.

Corollary 1.28. Let $k$ be an algebraically closed field.

(1) If $X$ is a connected quasi-projective curve with $N$ irreducible components, then $\mathrm{K}_{0}^{s g}(X) \simeq$ $\mathbb{Z}^{N-1}$. In particular, $\mathrm{K}_{0}^{s g}(X)=0$ if and only if $X$ is irreducible.

(2) If $X$ is a normal irreducible quasi-projective surface, then $\mathrm{K}_{0}^{s g}(X) \simeq \mathrm{Cl}(X) / \operatorname{Pic}(X)$. In particular, $\mathrm{K}_{0}^{s g}(X)=0$ if and only if $X$ is factorial.

Proof. We start by noticing that if $\operatorname{gr}^{i} \mathrm{~K}_{0}^{s g}(X)$ is the only nontrivial quotient of the topological filtration, then $\mathrm{K}_{0}^{s g}(X)=\operatorname{gr}^{i} \mathrm{~K}_{0}^{s g}(X)$. (1) follows as $\operatorname{gr}^{i} \mathrm{~K}_{0}^{s g}(X)$ are all zero except for $\operatorname{gr}^{0} \mathrm{~K}_{0}^{s g}(X)=\mathbb{Z}^{N-1}$, and similarly (2) follows using irreducibility of $X$ since $\operatorname{gr}^{i} \mathrm{~K}_{0}^{s g}(X)$ are all zero except for $\operatorname{gr}^{1} \mathrm{~K}_{0}^{s g}(X)=\mathrm{Cl}(X) / \operatorname{Pic}(X)$.

Recall functoriality of the singularity K-groups stated in Lemmas 1.14, 1.15, 1.16, We now explain how the topological filtration is affected by pull-back and push-forward. 
Lemma 1.29. Let $\phi: X \rightarrow Y$ be a morphism of finite Tor-dimension.

(1) If $\phi$ is flat or a regular closed embedding, then $\phi^{*}\left(F^{i} \mathrm{~K}_{0}^{s g}(Y)\right) \subset F^{i} \mathrm{~K}_{0}^{s g}(X)$.

(2) If $\phi$ is a vector bundle or an open embedding containing the singular locus of $Y$, then $\phi^{*}: F^{i} \mathrm{~K}_{0}^{s g}(Y) \simeq F^{i} \mathrm{~K}_{0}^{s g}(X)$.

(3) If $\phi$ is proper of codimension $c:=\operatorname{dim}(Y)-\operatorname{dim}(X)$, then $\phi_{*}\left(F^{i} \mathrm{~K}_{0}^{s g}(X)\right) \subset F^{i+c} \mathrm{~K}_{0}^{s g}(Y)$.

Proof. (1) The result in the case of flat morphisms follows from [33, Lemma 5.28], while in the case of regular embeddings it follows from [33, Theorem 5.27],

(2) The vector bundle case is [33, Lemma 5.29]. Let $\phi$ be an open embedding, since it is flat by (1) we have $\phi^{*}\left(F^{i} \mathrm{~K}_{0}^{s g}(Y)\right) \subset F^{i} \mathrm{~K}_{0}^{s g}(X)$ and we need to show that this is an equality. For that it suffices to show that every coherent sheaf $\mathcal{F}$ on $X$ with support in codimension $i$ can be extended to a coherent sheaf $\mathcal{F}^{\prime}$ on $Y$ with the same bound on support.

One constructs $\mathcal{F}^{\prime}$ as a coherent subsheaf of the quasi-coherent sheaf $\phi_{*}(\mathcal{F})$ (see [39, Ex. Chapter 2, 5.15]. Since $\phi_{*}(\mathcal{F})$ is supported on the closure of the support of $\mathcal{F}$, we see that $\mathcal{F}^{\prime}$ is supported in codimension $i$.

(3) If $\phi: X \rightarrow Y$ is a proper morphism of codimension $c$, then $\operatorname{Supp}\left(\phi_{*} \mathcal{E}\right) \subset \phi(\operatorname{Supp}(\mathcal{E}))$ and thus $\phi_{*}: F^{i} \mathrm{G}_{0}(X) \rightarrow F^{i+c} \mathrm{G}_{0}(Y)$ (see also [32], Ch. VI, Prop. 5.6) which implies the result.

We now explain how Knörrer periodicity (Theorem 1.3) shifts the topological filtration.

Proposition 1.30. The isomorphism $\mathrm{K}_{0}^{s g}\left(Z_{f}\right) \simeq \mathrm{K}_{0}^{s g}\left(Z_{g}\right)$ induced by Theorem 1.3 shifts the topological filtration by one, that is for all $i \geq 0$ we have natural isomorphisms $F^{i} \mathrm{~K}_{0}^{s g}\left(Z_{f}\right) \simeq$ $F^{i+1} \mathrm{~K}_{0}^{s g}\left(Z_{g}\right)$ and $\operatorname{gr}^{i} \mathrm{~K}_{0}^{s g}\left(Z_{f}\right) \simeq \operatorname{gr}^{i+1} \mathrm{~K}_{0}^{s g}\left(Z_{g}\right)$.

Proof. We know by Lemma 1.29 that $p^{*}$ preserves the topological filtration and that $i_{*}$ shifts it by one, however this only implies that $i_{*} p^{*}\left(F^{i} \mathrm{~K}_{0}^{s g}\left(Z_{f}\right)\right) \subset F^{i+1} \mathrm{~K}_{0}^{s g}\left(Z_{g}\right)$. To show the equality we give a different presentation of the Knörrer periodicity isomorphism.

Let $Y=B l_{Z_{f} \times 0}\left(X \times \mathbb{A}^{1}\right)$ be the blow up and let $E$ be the exceptional divisor. Since $Z_{f} \times \mathbb{A}^{1}$ is a complete intersection in $X \times \mathbb{A}^{1}$ the blow up enjoys the same properties which hold in the smooth case. For instance, $E$ is a projective bundle $\pi: E \rightarrow Z$, and there is a semiorthogonal decomposition [55], [10, Theorem 6.9]

$$
\mathcal{D}^{b}(Y)=\left\langle\mathcal{D}^{b}\left(Z_{f}\right), \mathcal{D}^{b}\left(X \times \mathbb{A}^{1}\right)\right\rangle .
$$

The inclusion of $\mathcal{D}^{b}\left(Z_{f}\right)$ into $\mathcal{D}^{b}(Y)$ is given by the fully faithful functor $\Phi: \mathcal{D}^{b}\left(Z_{f}\right) \rightarrow \mathcal{D}^{b}(Y)$

$$
\Phi(-)=i_{E *}\left(\mathcal{O}_{E}(-1) \otimes \pi^{*}(-)\right),
$$

and its left adjoint is

$$
\Psi(-)=\pi_{*}\left(\mathcal{O}_{E}(-1) \otimes i_{E}{ }^{*}(-)\right)[1] .
$$

Writing the open charts for the blow up one sees that one of the open charts is isomorphic to $Z_{g}$ while the other one is non-singular. We write $j: Z_{g} \rightarrow Y$ for the open embedding of the first open chart; on the level of singularity categories, $j^{*}$ is an equivalence by Proposition 1.2 . 
We consider the composition $j^{*} \Phi: \mathcal{D}^{b}\left(Z_{f}\right) \rightarrow \mathcal{D}^{b}\left(Z_{g}\right)$ and we now will show that $j^{*} \Phi=i_{*} p^{*}$. We note that restriction of $\pi$ to $E \cap Z_{g}$ is a trivial bundle so that $E \cap Z_{g}=Z_{f} \times \mathbb{A}^{1}$ (in fact $\pi$ itself is a trivial bundle since the normal bundle of $Z_{f} \times\{0\}$ in $X \times \mathbb{A}^{1}$ is trivial).

We consider the cartesian diagram

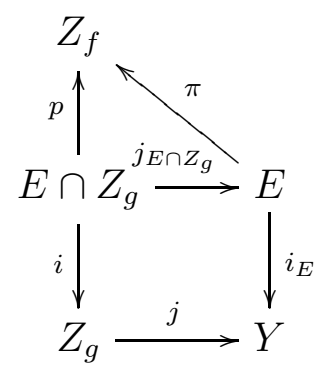

and by flat base change we compute that

$$
j^{*} \Phi(-)=j^{*} i_{E *}\left(\mathcal{O}_{E}(-1) \otimes \pi^{*}(-)\right)=i_{*}\left(j_{E \cap Z_{g}}^{*} \mathcal{O}_{E}(-1) \otimes j_{E \cap Z_{g}}^{*} \pi^{*}(-)\right)=i_{*} p^{*}(-) .
$$

where we used that $j_{E \cap Z_{g}}^{*} \mathcal{O}_{E}(-1) \simeq \mathcal{O}_{E \cap Z_{g}}$.

Now we check the effect of $j^{*} \Phi$ on the toplogical filtration. We rely on Lemma 1.29. Since $j^{*}$ strictly preserves the filtration it is sufficient to check that $\Phi$ strictly shifts the filtration by one: this holds true since $\pi^{*}$ preserves the filtration while $i_{E *}$ shifts it by one, and the left adjoint $\Psi$ of $\Phi$ which will become its inverse on the level of singularity categories, shifts the filtration by negative one: this holds since $i_{E}^{*}$ preserves the filtration while $\pi_{*}$ shifts it by negative one.

The next two examples consider the singularity Grothendieck group of split nodal affine quadrics, that is ordinary double points (cf [56, 3.3]).

Example 1.31 (Even-dimensional ordinary double points). Let $n=2 m$ and consider the split quadratic form

$$
q_{n}=\sum x_{i} y_{i}+z^{2} \in k\left[x_{1}, y_{1}, \ldots, x_{m}, y_{m}, z\right]
$$

and let $Q_{n} \subset \mathbb{A}^{n+1}$ be the $n$-dimensional nodal quadric defined by $q_{n}=0$.

From Knörrer periodicity we get

$$
\mathrm{K}_{0}^{s g}\left(Q_{n}\right) \simeq \mathrm{K}_{0}^{s g}\left(k[z] /\left(z^{2}\right)\right)=\mathbb{Z}_{2}
$$

see Example 1.21. Furthermore, since by dimension reasons the nonzero element of $\mathrm{K}_{0}^{s g}\left(k[z] /\left(z^{2}\right)\right)$ has support in codimension zero, by the shift of the topological filtration of Proposition 1.30 we get

$$
\mathrm{K}_{0}^{s g}\left(Q_{n}\right)=\operatorname{gr}^{n / 2} \mathrm{~K}_{0}^{s g}\left(Q_{n}\right)=\mathbb{Z}_{2} .
$$

Explicitly $\mathrm{K}_{0}^{s g}\left(Q_{n}\right)$ can be seen to be generated by the structure sheaf of $n / 2$-codimensional subvariety $V\left(y_{1}, \ldots, y_{m}, z\right) \subset Q_{n}$. 
Example 1.32 (Odd-dimensional ordinary double points). Let $n=2 m-1$ and consider

$$
q_{n}=\sum x_{i} y_{i} \in k\left[x_{1}, y_{1}, \ldots, x_{m}, y_{m}\right]
$$

and let $Q_{n} \subset \mathbb{A}^{n+1}$ be the $n$-dimensional nodal quadric defined by $q_{n}=0$.

By Knörrer periodicity we get

$$
\mathrm{K}_{0}^{s g}\left(Q_{n}\right) \simeq \mathrm{K}_{0}^{s g}(k[x, y] /(x y))
$$

and the latter Grothendieck group is isomorphic to $\mathbb{Z}$ by Corollary 1.28 (1), generated by the structure sheaves of one of the two irreducible components (cf Example 1.23). Using Proposition 1.30 we obtain

$$
\mathrm{K}_{0}^{s g}\left(Q_{n}\right)=\operatorname{gr}^{(n-1) / 2} \mathrm{~K}_{0}^{s g}\left(Q_{n}\right)=\mathbb{Z},
$$

generated by the structure sheaf of a codimension $(n-1) / 2$ linear space $V\left(y_{1}, \ldots, y_{m}\right) \subset Q_{n}$.

\section{Singularity K-Theory of QUOTIENT SINGUlarities}

2.1. The local case: non-positive K-groups. For a finite group $G \subset \mathrm{GL}_{n}(k)$ we consider the quotient variety $\mathbb{A}^{n} / G=\operatorname{Spec}\left(k\left[x_{1}, \ldots, x_{n}\right]^{G}\right)$. In this subsection we study $\mathrm{G}_{0}\left(\mathbb{A}^{n} / G\right)$ as well as $\mathrm{K}$-groups $\mathrm{K}_{i}\left(\mathbb{A}^{n} / G\right)$ and $\mathrm{K}_{i}^{s g}\left(\mathbb{A}^{n} / G\right)$ for $i \leq 0$.

Proposition 2.1. Assume that $\mathbb{A}^{n} / G$ has an isolated singularity at the origin. Then

$$
\mathrm{K}_{0}\left(\mathbb{A}^{n} / G\right) \simeq \mathbb{Z} \quad \text { and } \mathrm{K}_{-j}\left(\mathbb{A}^{n} / G\right)=\mathrm{K}_{-j}^{s g}\left(\mathbb{A}^{n} / G\right)=0 \text { for } j>0 .
$$

Proof. Since $k\left[x_{1}, \ldots, x_{n}\right]^{G}$ is a positively graded algebra, we can use [20, Theorem 1.2] to express non-positive $K$-theory groups as follows:

$$
\mathrm{K}_{0}\left(\mathbb{A}^{n} / G\right)=\mathbb{Z} \oplus \operatorname{Pic}\left(\mathbb{A}^{n} / G\right) \oplus \bigoplus_{i=1}^{n-1} H_{\mathrm{cdh}}^{i}\left(\mathbb{A}^{n} / G, \Omega_{/ \mathbb{Q}}^{i}\right) / d H_{\mathrm{cdh}}^{i}\left(\mathbb{A}^{n} / G, \Omega_{/ \mathbb{Q}}^{i-1}\right)
$$

and for $j>0$

$$
\mathrm{K}_{-j}\left(\mathbb{A}^{n} / G\right)=H_{\mathrm{cdh}}^{j}\left(\mathbb{A}^{n} / G, \mathcal{O}\right) \oplus \bigoplus_{i=1}^{n-j-1} H_{\mathrm{cdh}}^{i+j}\left(\mathbb{A}^{n} / G, \Omega_{/ \mathbb{Q}}^{i}\right) / d H_{\mathrm{cdh}}^{i+j}\left(\mathbb{A}^{n} / G, \Omega_{/ \mathbb{Q}}^{i-1}\right) .
$$

Here $H_{\text {cdh }}^{*}$ denotes cohomology of $\mathbb{A}^{n} / G$ defined via the cdh-topology on Sch $/ k$ [68] and the group $d H_{\text {cdh }}^{j}\left(\mathbb{A}^{n} / G, \Omega_{/ \mathbb{Q}}^{i-1}\right)$ is the image of the map $d: H_{\text {cdh }}^{j}\left(\mathbb{A}^{n} / G, \Omega_{/ \mathbb{Q}}^{i-1}\right) \rightarrow H_{\mathrm{cdh}}^{j}\left(\mathbb{A}^{n} / G, \Omega_{/ \mathbb{Q}}^{i}\right)$ induced by the Kähler differential.

Let us show that cohomology groups $H^{q}\left(\mathbb{A}^{n} / G, \Omega_{/ \mathbb{Q}}^{p}\right)$ are zero for all $q>0, p \geq 0$. Since every $G$-representation is defined over a subfield $k_{0} \subset k$ which is a finite extension of $\mathbb{Q}, \mathbb{A}^{n} / G$ admits a model over $k_{0}$. 
Let us show that for every variety $X / k$ admitting a model $X_{0} / k_{0}$ we have the Künneth formula for cdh cohomology groups (cf. [18, Corollary 4.5]):

$$
H_{\mathrm{cdh}}^{q}\left(X, \Omega_{/ \mathbb{Q}}^{p}\right) \simeq \bigoplus_{p=i+j} H_{\mathrm{cdh}}^{q}\left(X, \Omega_{/ k}^{i}\right) \otimes_{k} \Omega_{k / \mathbb{Q}}^{j}
$$

for $p, q \geq 0$.

Let us first assume that $X$ is smooth. In this case using [21, Corollary 2.5], (2.1) is equivalent to the isomorphism of Zariski cohomology groups

$$
H^{q}\left(X, \Omega_{X / \mathbb{Q}}^{p}\right) \simeq \bigoplus_{p=i+j} H^{q}\left(X, \Omega_{X / k}^{i}\right) \otimes_{k} \Omega_{k / \mathbb{Q}}^{j}
$$

Note that as $k_{0}$ is finite separable over $\mathbb{Q}$ we may replace $\mathbb{Q}$ by $k_{0}$ in (2.2). Since $X$ has a model over $k_{0}$ we have a splitting $\Omega_{X / k_{0}}^{1} \simeq \Omega_{X / k}^{1} \oplus\left(\Omega_{k / k_{0}}^{1} \otimes \mathcal{O}_{X}\right)$ and hence

$$
\Omega_{X / k_{0}}^{p} \simeq \bigoplus_{p=i+j} \Omega_{X / k}^{i} \otimes \Omega_{k / k_{0}}^{j} .
$$

Applying cohomology one obtains (2.2) and hence (2.1) in the smooth case.

In general, that is when $X$ is singular, (2.1) follows by induction on dimension using the cdh descent exact sequence [68, 12.1] applied to a resolution of $X$ definable over $k_{0}$.

Finally, by [19, Lemma 2.1] and [24, Theorem 5.12] we see that

$$
H_{\mathrm{cdh}}^{q}\left(\mathbb{A}^{n} / G, \Omega_{/ k}^{p}\right) \simeq H^{q}\left(\mathbb{A}^{n}, \Omega_{\mathbb{A}^{n} / k}^{p}\right)^{G},
$$

and since $\mathbb{A}^{n}$ is affine, these cohomology groups vanish for $q>0$. So combining everything together we get $\mathrm{K}_{-j}\left(\mathbb{A}^{n} / G\right)=0$ and $\mathrm{K}_{0}\left(\mathbb{A}^{n} / G\right)=\mathbb{Z} \oplus \operatorname{Pic}\left(\mathbb{A}^{n} / G\right)=\mathbb{Z}$, since the Picard group of a normal graded $k$-algebra is zero.

Remark 2.2. In the previous version of this paper we claimed that every vector bundle on $\mathbb{A}^{n} / G$ is trivial. We do not know if this statement is true. We thank Sasha Kuznetsov for pointing out an error in our argument.

Corollary 2.3. If $\mathbb{A}^{n} / G$ is an isolated singularity, then the singularity category $\mathcal{D}^{\mathrm{sg}}\left(\mathbb{A}^{n} / G\right)$ is idempotent complete.

Proof. As $\mathrm{K}_{-1}\left(\mathbb{A}^{n} / G\right)=0$ by Proposition 2.1, the result follows from Lemma 1.11.

Remark 2.4. It is not true that every affine quasi-homogeneous or $\mathbb{A}^{1}$-contractible singularity has an idempotent complete singularity category: the simplest example is provided by the socalled Bloch-Murthy surface singularity $X$ given by $x^{2}+y^{3}+z^{7}=0$ which has non-vanishing $K_{-1}(X)$ [76, Example 6.1].

Proposition 2.5. Let $G$ be a finite group acting linearly on the affine space $\mathbb{A}^{n}$ over a field $k$. Then we have

$$
\mathrm{G}_{0}\left(\mathbb{A}^{n} / G\right)=\mathbb{Z} \oplus \mathrm{K}_{0}^{s g}\left(\mathbb{A}^{n} / G\right)
$$


and $\mathrm{K}_{0}^{s g}\left(\mathbb{A}^{n} / G\right)$ is a finite torsion group.

Proof. By Proposition 1.20 (3) there is a split short exact sequence

$$
0 \rightarrow \mathbb{Z} \rightarrow \mathrm{G}_{0}\left(\mathbb{A}^{n} / G\right) \rightarrow \mathrm{K}_{0}^{s g}\left(\mathbb{A}^{n} / G\right) \rightarrow 0
$$

where the first map is split by the rank map. Let us show that $\mathrm{K}_{0}^{s g}\left(\mathbb{A}^{n} / G\right)$ is finite torsion. The fact that $\mathrm{G}_{0}\left(\mathbb{A}^{n} / G\right)$ is finitely-generated is well-known [3] and follows e.g. from the fact that the push-forward functor from the equivariant category to the category of coherent sheaves on the quotient variety $\pi_{*}: \mathcal{D}_{G}^{b}\left(\mathbb{A}^{n}\right) \rightarrow \mathcal{D}^{b}\left(\mathbb{A}^{n} / G\right)$ is essentially surjective by Theorem 2.30 (1). Thus it suffices to show that $\mathrm{G}_{0}\left(\mathbb{A}^{n} / G\right)$ is of rank one. For that we can work rationally and compare $\mathrm{G}_{0}$ to the Chow groups. We have a chain of isomorphisms

$$
\mathrm{G}_{0}\left(\mathbb{A}^{n} / G\right) \otimes \mathbb{Q} \simeq \mathrm{CH}_{*}\left(\mathbb{A}^{n} / G\right) \otimes \mathbb{Q} \simeq \mathrm{CH}_{*}\left(\mathbb{A}^{n}\right)^{G} \otimes \mathbb{Q} \simeq \mathbb{Q}
$$

where the first isomorphism is the Grothendieck-Riemann-Roch Theorem for singular varieties [5, Chapter III] and the second isomorphism is [31, Example 1.7.6]. We conclude that $\mathrm{G}_{0}\left(\mathbb{A}^{n} / G\right)$ is a finitely generated abelian group of rank one and that $\mathrm{K}_{0}^{s g}\left(\mathbb{A}^{n} / G\right)$ is finite torsion.

We call an element $g \in \mathrm{GL}_{n}(k)$ a reflection if $g$ has finite order and acts trivially on a hyperplane. We need the following well-known Lemmas.

Lemma 2.6 ([7, Theorem 3.9.2]). Let $G$ be a finite subgroup of $\mathrm{GL}_{n}(k)$ and let $N$ be the subgroup of $G$ generated by reflections. There is a natural isomorphism $\operatorname{Cl}\left(\mathbb{A}^{n} / G\right) \simeq \widehat{G / N}$.

Proof. Our proof relies on equivariant Chow groups [25]. Let us first assume that $G$ does not contain reflections. In this case there is a $G$-invariant subvariety $Z \subset \mathbb{A}^{n}$ of codimension at least two, such that $G$ acts freely on $\mathbb{A}^{n} \backslash Z$. Let $\pi: \mathbb{A}^{n} \rightarrow \mathbb{A}^{n} / G$ be the quotient map. Since removing locus of codimension two does not change $(n-1)$-st Chow groups, we have a chain of isomorphisms

$$
\mathrm{Cl}\left(\mathbb{A}^{n} / G\right)=\mathrm{CH}_{n-1}\left(\mathbb{A}^{n} / G\right) \simeq \mathrm{CH}_{n-1}\left(\mathbb{A}^{n} / G \backslash \pi(Z)\right)=\mathrm{CH}_{n-1}\left(\left(\mathbb{A}^{n} \backslash Z\right) / G\right) \simeq \mathrm{CH}_{n-1}^{G}\left(\mathbb{A}^{n}\right) .
$$

Since $\mathbb{A}^{n}$ is smooth, we have $\mathrm{CH}_{n-1}^{G}\left(\mathbb{A}^{n}\right)=\operatorname{Pic}^{G}\left(\mathbb{A}^{n}\right)$, and the latter group of $G$-equivariant line bundles on $\mathbb{A}^{n}$ is isomorphic to the group $\widehat{G}$ of characters of $G$.

In the general case the fixed locus of the action of $G / N$ on $\mathbb{A}^{n} / N \simeq \mathbb{A}^{n}$ does not contain divisors and the same argument applies to show that $\mathrm{Cl}\left(\mathbb{A}^{n} / G\right) \simeq \widehat{G / N}$.

Lemma 2.7. For every $0 \leq i \leq n-1, \mathrm{CH}_{i}\left(\mathbb{A}^{n} / G\right)$ is annihilated by $|G|$.

Proof. Let $V \subset \mathbb{A}^{n} / G$ be a subvariety, let $\pi^{-1}(V)$ be the scheme theoretic preimage of $V$ under the quotient morphism $\pi: \mathbb{A}^{n} \rightarrow \mathbb{A}^{n} / G$, and let $W$ be a reduced irreducible component of $\pi^{-1}(V)$. Let us show that the degree of the field extension $[k(W): k(V)]$ divides $|G|$.

Since we assume $k$ to be of characteristic zero, $G$ is a linearly reductive $k$-group scheme. Thus according to [29, Proof of Theorem 1.1, p. 28], the $G$-invariant ring of $A:=k\left[x_{1}, \ldots, x_{n}\right] \otimes_{k\left[\mathbb{A}^{n} / G\right]}$ 
$k(V)$ is just $k(V)$. If $A_{r e d}$ is the quotient of $A$ by the nilradical, then

$$
A_{\text {red }}=\prod_{i=1}^{r} k\left(W_{i}\right)
$$

where $W_{1}, \ldots, W_{r}$ are all components of $\pi^{-1}(V)$. The action of $G$ on $A$ induces an action on $A_{\text {red }}$, and $A_{r e d}^{G}=A^{G}=k(V)$. Since $G$ acts on the components $W_{1}, \ldots, W_{r}$ transitively, the degrees $\left[k\left(W_{i}\right): k(V)\right]$ are equal to each other, and it is easy to see that they divide $|G|$.

Since $\mathrm{CH}_{i}\left(\mathbb{A}^{n}\right)=0$ in the considered range, by definition of push-forward on Chow groups we get

$$
0=\pi_{*}([W])=[k(W): k(V)] \cdot[V]
$$

so that $[V] \in \mathrm{CH}_{i}\left(\mathbb{A}^{n} / G\right)$ is $|G|$-torsion.

Proposition 2.8. There is a well-defined surjective first Chern class homomorphism

$$
c_{1}: \mathrm{K}_{0}^{s g}\left(\mathbb{A}^{n} / G\right) \rightarrow \widehat{G / N} .
$$

If $n=2$ and $k$ is algebraically closed, then $c_{1}$ is an isomorphism.

Proof. We claim that there is a Grothendieck-Riemann-Roch without denominators style surjection

$$
\left(\mathrm{rk}, c_{1}\right): \mathrm{G}_{0}\left(\mathbb{A}^{n} / G\right) \rightarrow \mathbb{Z} \oplus \mathrm{CH}_{n-1}\left(\mathbb{A}^{n} / G\right) .
$$

Indeed, since $\mathbb{A}^{n} / G$ is normal, to construct $c_{1}$ one may simply remove the singular locus of $\mathbb{A}^{n} / G$ and thus reduce to the smooth case, and the surjectivity follows easily.

Splitting off the direct summand $\mathbb{Z}$ corresponding to the trivial bundles and using Proposition 2.5 and Lemma 2.6 we get the desired surjection.

By construction of the topological filtration on $\mathrm{K}_{0}^{s g}(X)$ we have $\operatorname{Ker}\left(c_{1}\right)=F^{2} \mathrm{~K}_{0}^{s g}(X)$ (cf. proof of Proposition $1.24(2))$, in particular if $n=2$, then $\operatorname{Ker}\left(c_{1}\right)=F^{2} \mathrm{~K}_{0}^{s g}(X)=0$ by Proposition 1.24 (4).

Proposition 2.9. Let $k$ be an algebraically closed field of characteristic zero. Then every element of $\mathrm{K}_{0}^{s g}\left(\mathbb{A}^{n} / G\right)$ is annihilated by $|G|^{n-1}$.

Proof. We consider the topological filtration on $\mathrm{K}_{0}^{s g}\left(\mathbb{A}^{n} / G\right)$ and its associated graded pieces $\operatorname{gr}^{i} \mathrm{~K}_{0}^{s g}\left(\mathbb{A}^{n} / G\right)$. By Proposition 1.24 we have $\operatorname{gr}^{0} \mathrm{~K}_{0}^{s g}\left(\mathbb{A}^{n} / G\right)=\operatorname{gr}^{n} \mathrm{~K}_{0}^{s g}\left(\mathbb{A}^{n} / G\right)=0$ so that the filtration has the form

$$
0=F^{n} \mathrm{~K}_{0}^{s g}\left(\mathbb{A}^{n} / G\right) \subset F^{n-1} \mathrm{~K}_{0}^{s g}\left(\mathbb{A}^{n} / G\right) \subset \ldots \subset F^{1} \mathrm{~K}_{0}^{s g}\left(\mathbb{A}^{n} / G\right)=\mathrm{K}_{0}^{s g}\left(\mathbb{A}^{n} / G\right) .
$$

By Proposition 1.24, each subquotient $\operatorname{gr}^{i} \mathrm{~K}_{0}^{s g}\left(\mathbb{A}^{n} / G\right), 1 \leq i \leq n-1$, admits a surjection $\mathrm{CH}_{n-i}\left(\mathbb{A}^{n} / G\right) \rightarrow \operatorname{gr}^{i} \mathrm{~K}_{0}^{s g}\left(\mathbb{A}^{n} / G\right)$ and by Lemma 2.7, each of these groups is annihilated by $|G|$. This means that multiplication by $|G|$ shifts the filtration: $|G| \cdot F^{i} \mathrm{G}_{0}\left(\mathbb{A}^{n} / G\right) \subset F^{i+1} \mathrm{G}_{0}\left(\mathbb{A}^{n} / G\right)$, in particular multiplication by $|G|^{n-1}$ acts as the zero map. 
The next proposition gives the formula for $\mathrm{K}_{0}^{s g}\left(\mathbb{A}^{n} / G\right)$ in the isolated singularity case. For other approaches to how to compute this group see [3], [49], [40].

Proposition 2.10 (34]). Let $G$ be a finite group acting linearly on $\mathbb{A}^{n}$ such that the $G$-action on $\mathbb{A}^{n} \backslash\{0\}$ is free. Let $\rho$ be the corresponding representation of $G$. Then we have

$$
\mathrm{G}_{0}\left(\mathbb{A}^{n} / G\right) \simeq R(G) / r R(G)
$$

where $R(G)$ is the representation ring of $G$ and $r \in R(G)$ is the Koszul class

$$
r=\sum_{i=0}^{n}(-1)^{i}\left[\Lambda^{i}\left(\rho^{\vee}\right)\right] .
$$

Proof. The proof uses equivariant algebraic K-theory [71]. Since $G$ acts freely away from 0, there is an isomorphism

$$
\mathrm{K}_{0}^{G}\left(\mathbb{A}^{n} \backslash\{0\}\right) \simeq \mathrm{G}_{0}\left(\frac{\mathbb{A}^{n} \backslash\{0\}}{G}\right) .
$$

Let $i:\{0\} \rightarrow \mathbb{A}^{n}, \bar{i}:\{0\} \rightarrow \mathbb{A}^{n} / G$ be the closed embeddings. Consider the localization exact sequences of $\mathrm{G}_{0}$ and $\mathrm{K}_{0}^{G}$ :

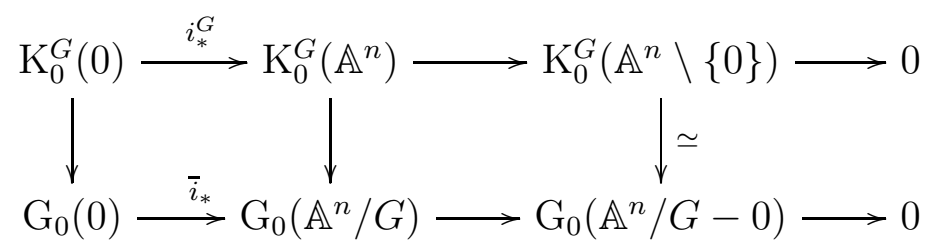

related by push-forward maps followed by taking $G$-invariants. The push-forward $\bar{i}_{*}$ is a zero map since it factor through $i_{*}=0$. By equivariant homotopy invariance [71, 4.1] we have an isomorphism $\mathrm{K}_{0}^{G}\left(\mathbb{A}^{n} / G\right) \simeq \mathrm{K}_{0}^{G}(0) \simeq R(G)$ and under these identifications the push-forward $i_{*}^{G}: \mathrm{K}_{0}^{G}(0) \rightarrow \mathrm{K}_{0}^{G}\left(\mathbb{A}^{n}\right)$ corresponds to the multiplication by the class $\left[\mathcal{O}_{0}\right]=r$.

Putting everything together we obtain

$$
\mathrm{G}_{0}\left(\mathbb{A}^{n} / G\right) \simeq \mathrm{G}_{0}\left(\left(\mathbb{A}^{n} \backslash\{0\}\right) / G\right) \simeq R(G) / r R(G) .
$$

Remark 2.11. If $G$ has no reflections, then the condition that $G$ acts freely on $\mathbb{A}^{n} \backslash\{0\}$ is equivalent to the quotient $\mathbb{A}^{n} / G$ to have an isolated singularity at the origin.

Remark 2.12. Since $\mathrm{K}_{0}^{s g}\left(\mathbb{A}^{n} / G\right)$ is a finite group by Proposition 2.5 we see that under the assumptions of Proposition 2.10 the linear map $r: R(G) \rightarrow R(G)$ has cokernel of rank one, and so it has a one-dimensional kernel.

Example 2.13 ([34]). Computing $R(G) / r R(G)$ for a two-dimensional ADE singularity $\mathbb{A}^{2} / G$, one can compute $\mathrm{K}_{0}^{\text {sg }}\left(\mathbb{A}^{2} / G\right)$ using Proposition 2.10 as follows: 


\begin{tabular}{|c|c|}
\hline Type & $\mathrm{K}_{0}^{s g}\left(\mathbb{A}^{2} / G\right)$ \\
\hline \hline$A_{n}$ & $\mathbb{Z} /(n+1) \mathbb{Z}$ \\
\hline$D_{n}, n$ even & $\mathbb{Z} / 2 \mathbb{Z} \times \mathbb{Z} / 2 \mathbb{Z}$ \\
\hline$D_{n}, n$ odd & $\mathbb{Z} / 4 \mathbb{Z}$ \\
\hline$E_{6}$ & $\mathbb{Z} / 3 \mathbb{Z}$ \\
\hline$E_{7}$ & $\mathbb{Z} / 2 \mathbb{Z}$ \\
\hline$E_{8}$ & 0 \\
\hline
\end{tabular}

(note a typo in [34] in the $E_{7}$ case on page 415). The same result can be obtained using Proposition [2.8, and another way is given by Yoshino using Auslander-Reiten sequences [78, Chapter 13].

Corollary 2.14. Let $k$ be an algebraically closed field of characteristic zero and let $X$ be the local $\frac{1}{m}(\overbrace{1, \ldots, 1}^{n})$ singularity, that is $X=\mathbb{A}^{n} / \mathbb{Z}_{m}$ with the diagonal action by the primitive root of unity. Then $\mathrm{K}_{0}^{s g}(X)$ is a finite abelian group of order $m^{n-1}$.

Proof. Let us fix a primitive character $\rho$ of $\mathbb{Z}_{m}$. Then the representation ring is $R\left(\mathbb{Z}_{m}\right)=$ $\mathbb{Z}[x] /\left(x^{m}-1\right)$ where we choose $x$ to be the class $\left[\rho^{\vee}\right]$. Then

$$
r=\sum_{i=0}^{n}(-1)^{i}\left[\Lambda^{i}\left(\rho^{\vee}\right)\right]=\sum_{i=0}^{n}\left(\begin{array}{c}
n \\
i
\end{array}\right)(-1)^{i} x^{i}=(1-x)^{n} \in R(G)
$$

and after making a substitution $y=1-x$, we obtain

$$
\mathrm{G}_{0}(X)=\mathbb{Z}[y] /\left(y^{n}, m y-\left(\begin{array}{c}
m \\
2
\end{array}\right) y^{2}+\ldots\right)=\mathbb{Z} \cdot 1 \oplus \mathrm{K}_{0}^{s g}(X)
$$

so that $\mathrm{K}_{0}^{s g}(X)$ is a quotient of a free $\mathbb{Z}$-module with the basis $y, y^{2}, \ldots, y^{n-1}$ by the uppertriangular relations $m y^{i}-\left(\begin{array}{c}m \\ 2\end{array}\right) y^{i+1}+\ldots$ for $i \geq 1$. This means that $\mathrm{K}_{0}^{s g}(X)$ is a finite abelian group of order $m^{n-1}$.

We abuse the notation slightly by writing $X=\frac{1}{m}(1, \ldots, 1)$ for the corresponding local singularity. The precise structure of $\mathrm{K}_{0}^{s g}\left(\frac{1}{m}(\overbrace{1, \ldots, 1}^{n})\right)$ will vary depending on $m$ and $n$.

Example 2.15. For $n=2$ we have $\mathrm{K}_{0}^{s g}\left(\frac{1}{m}(1,1)\right) \simeq \mathbb{Z}_{m}$, in accordance with Proposition [2.9.

Example 2.16. For $n=3$ one can see that

$$
\mathrm{K}_{0}^{s g}\left(\frac{1}{m}(1,1,1)\right)= \begin{cases}\left(\mathbb{Z}_{m}\right)^{2}, & m \text { odd } \\ \mathbb{Z}_{m / 2} \times \mathbb{Z}_{2 m}, & m \text { even }\end{cases}
$$

Example 2.17. If $m=2$ and $n$ is arbitrary, one can see that $\mathrm{K}_{0}^{s g}\left(\mathbb{A}^{n} / \mathbb{Z}_{2}\right) \simeq \mathbb{Z}_{2^{n-1}}$ (here the action of $\mathbb{Z}_{2}$ on $\mathbb{A}^{n}$ is $\left.v \mapsto-v\right)$. 


\subsection{The local case: positive K-groups.}

Proposition 2.18. Let $k$ be an algebraically closed field of characteristic zero, and let $G \subset$ $\mathrm{GL}_{n}(k)$ be a finite group such that the $G$-action on $\mathbb{A}^{n} \backslash\{0\}$ is free. For every $j \geq 0$ consider the group $T_{j}=\operatorname{Tor}\left(\mathrm{K}_{0}^{s g}\left(\mathbb{A}^{n} / G\right), \mathrm{K}_{j}(k)\right)$.

(1) $T_{j}$ is a finite torsion group annihilated by $|G|^{n-1}$, and $T_{j}=0$ for all even $j$.

(2) For every $j \geq 1$ there is a short exact sequence

$$
0 \rightarrow \mathrm{K}_{j}(k) \rightarrow \mathrm{G}_{j}\left(\mathbb{A}^{n} / G\right) \rightarrow T_{j-1} \rightarrow 0,
$$

where the first map is the pull-back from $\operatorname{Spec}(k)$. In particular for all $j \geq 1$ we have $G_{j}\left(\mathbb{A}^{n} / G\right) \otimes$ $\mathbb{Z}[1 /|G|] \simeq \mathrm{K}_{j}(k) \otimes \mathbb{Z}[1 /|G|]$ and for all odd $j \geq 1$ we have $\mathrm{G}_{j}\left(\mathbb{A}^{n} / G\right) \simeq \mathrm{K}_{j}(k)$.

(3) For every $j \geq 1$, there is an exact sequence

$$
0 \rightarrow T_{j-1} \rightarrow \mathrm{K}_{j}^{s g}\left(\mathbb{A}^{n} / G\right) \rightarrow \mathrm{K}_{j-1}\left(\mathbb{A}^{n} / G\right) \rightarrow \mathrm{K}_{j-1}(k) \rightarrow 0,
$$

where the last morphism in the sequence is induced by restriction to the rational point $0 \in \mathbb{A}^{n} / G$.

We prove the Proposition at the end of this subsection.

Corollary 2.19. If $\mathbb{A}^{n} / G$ is an isolated singularity over an algebraically closed field of characteristic zero then $\mathrm{K}_{1}^{s g}\left(\mathbb{A}^{n} / G\right)=0$.

Proof. We may assume that $G$ acts freely on $\mathbb{A}^{n} \backslash\{0\}$ (see Remark 2.11 and proof of Proposition 2.8). The result follows from (2.5) using the fact that $T_{0}=0$ and Proposition 2.1 which says that $\mathrm{K}_{0}\left(\mathbb{A}^{n} / G\right)=\mathrm{K}_{0}(k)=\mathbb{Z}$.

Remark 2.20. For non-algebraically closed field, see Example 3.9. We do not know if $\mathrm{K}_{1}^{s g}\left(\mathbb{A}^{n} / G\right)=$ 0 in the non-isolated singularity case.

Remark 2.21. The structure of the groups $\mathrm{K}_{j}\left(\mathbb{A}^{n} / G\right)$ for $j \geq 1$ is in general not known. Since the work of Srinivas it is known that $\operatorname{Ker}\left(\mathrm{K}_{1}\left(\mathbb{A}^{n} / G\right) \rightarrow \mathrm{K}_{1}(k)\right)$ is "huge", that is as large as the base field $k$, even in the simplest $\frac{1}{2}(1,1)$ case [65], and the same follows for $\mathrm{K}_{2}^{\text {sg }}\left(\mathbb{A}^{n} / G\right)$ from Proposition 2.18.

In order to prove Proposition [2.18, we use the language of equivariant K-theory [71] which for finite groups can also be interpreted as K-theory of Deligne-Mumford stacks [42].

Lemma 2.22. In the assumptions of Proposition 2.18, let $i: \operatorname{Spec}(k) \rightarrow \mathbb{A}^{n}$ be the closed embedding of the origin 0 . Then the following is true. 
(1) We have natural $R(G)$-module isomorphisms $K_{j}^{G}\left(\mathbb{A}^{n}\right) \stackrel{i_{G}^{*}}{\simeq} K_{j}^{G}(k) \simeq R(G) \otimes K_{j}(k)$ and a commutative diagram

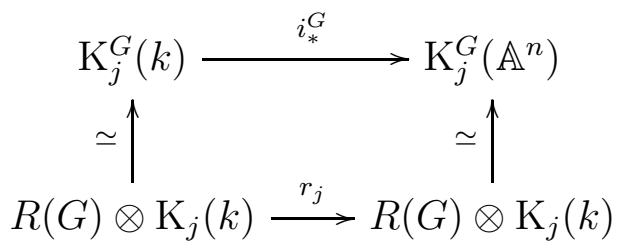

where $r_{j}$ is multiplication by the Koszul class $r \in R(G)$ defined in Proposition 2.10.

(2) Let $\pi_{0}$ be the projection from the Deligne-Mumford stack $[\operatorname{Spec}(k) / G]$ to its coarse moduli space $\operatorname{Spec}(k)$ and let $\alpha_{j}$ be the restriction of $\pi_{0, *}: \mathrm{K}_{j}^{G}(k) \rightarrow \mathrm{K}_{j}(k)$ to $\operatorname{Ker}\left(i_{*}^{G}\right)=\operatorname{Ker}\left(r_{j}\right)$. Then for every $j \geq 0$ there is a commutative diagram

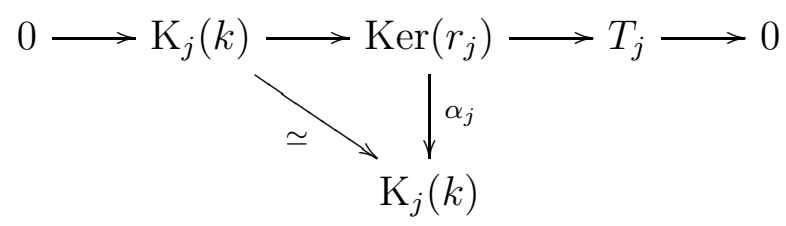

where $T_{j}$ is defined as in Proposition 2.18 and the top row is exact.

(3) Let $\pi$ be the projection from the Deligne-Mumford stack $\left[\mathbb{A}^{n} / G\right]$ to its coarse moduli space $\mathbb{A}^{n} / G$. For every $j \geq 0$ consider the subgroup $\mathrm{K}_{j}(k) \simeq 1 \otimes \mathrm{K}_{j}(k) \subset R(G) \otimes \mathrm{K}_{j}(k)=$ $\mathrm{K}_{j}^{G}\left(\mathbb{A}^{n}\right)$, and let $\beta_{j}$ be the restriction of $\pi_{*}: \mathrm{K}_{j}^{G}\left(\mathbb{A}^{n}\right) \rightarrow \mathrm{G}_{j}\left(\mathbb{A}^{n} / G\right)$ to this subgroup. Then $\beta_{j}$ is isomorphic to pullback morphism $p^{*}: \mathrm{K}_{j}(k) \rightarrow \mathrm{G}_{j}\left(\mathbb{A}^{n} / G\right)$ induced by the structure morphism $p: \mathbb{A}^{n} / G \rightarrow \operatorname{Spec}(k)$.

Furthermore, for $j \geq 1$ the embedding $1 \otimes \mathrm{K}_{j}(k) \subset R(G) \otimes K_{j}(k)$ induces an isomorphism $\mathrm{K}_{j}(k) \simeq \operatorname{Coker}\left(r_{j}\right)$.

Proof. (1) $i_{G}^{*}$ being an isomorphism is the standard homotopy invariance of $K$-theory in the regular case [71, 4.1], $\mathrm{K}_{j}^{G}(k) \simeq R(G) \otimes \mathrm{K}_{j}(k)$ holds e.g. by [75, Proposition 1.6]. The commutative diagram follows from [75, Lemma 1.7].

(2) Since the map $r_{j}$ is isomorphic to $r \otimes \mathrm{id}, \operatorname{Ker}\left(r_{j}\right)$ can be computed via the Universal Coefficient Theorem applied to the complex $[r: R(G) \rightarrow R(G)]$ as follows. We have

$$
0 \rightarrow \operatorname{Ker}(r) \otimes K_{j}(k) \rightarrow \operatorname{Ker}\left(r_{j}\right) \rightarrow \operatorname{Tor}\left(\operatorname{Coker}(r), K_{j}(k)\right) \rightarrow 0 .
$$

By Remark 2.12, $\operatorname{Ker}(r)=\mathbb{Z} \cdot t$, for some element $t \in R(G)$, and by Proposition 2.10, Coker $(r) \simeq$ $\mathbb{Z} \oplus \mathrm{K}_{0}^{s g}\left(\mathbb{A}^{n} / G\right)$. We see that $\operatorname{Ker}(r) \otimes \mathrm{K}_{j}(k)=\mathrm{K}_{j}(k)$ and

$$
\left.\operatorname{Tor}\left(\operatorname{Coker}(r), K_{j}(k)\right)=\operatorname{Tor}\left(\mathrm{K}_{0}^{s g}\left(\mathbb{A}^{n} / G\right)\right), \mathrm{K}_{j}(k)\right)=T_{j}
$$

so that the top row of (2.6) is exact. We compute $\alpha_{j}$ as follows

$$
\left.\alpha_{j}\right|_{t \otimes \mathrm{K}_{j}(k)}=\left.\pi_{0, *}\right|_{t \otimes \mathrm{K}_{j}(k)}=\pi_{0, *}(t) \cdot \mathrm{id}_{\mathrm{K}_{j}(k)},
$$


and for commutativity of (2.6) it remains to show that $\pi_{0, *}(t)= \pm 1$. This follows easily by extending the commutative diagram (2.3) on term to the left [71, Theorem 2.7] (cf $j=1$ case in (2.7) in the Proof of Proposition 2.18).

(3) The fact that $\beta_{j}$ is equal to $p^{*}$ follows from the projection formula. Since tensor product is right exact we have

$$
\operatorname{Coker}\left(r_{j}\right)=\operatorname{Coker}(r) \otimes \mathrm{K}_{j}(k) \simeq\left(\mathbb{Z} \oplus \mathrm{K}_{0}^{s g}\left(\mathbb{A}^{n} / G\right)\right) \otimes \mathrm{K}_{j}(k) .
$$

Since $k$ is algebraically closed, by a result of Suslin [67], for every $j \geq 1, \mathrm{~K}_{j}(k)$ is a divisible group, so that since $\mathrm{K}_{0}^{s g}\left(\mathbb{A}^{n} / G\right)$ is torsion, $\left.\mathrm{K}_{0}^{s g}\left(\mathbb{A}^{n} / G\right)\right) \otimes \mathrm{K}_{j}(k)=0$, and we have

$$
\operatorname{Coker}\left(r_{j}\right) \simeq \mathrm{K}_{j}(k),
$$

induced by tensoring $\mathrm{rk}: R(G) \rightarrow \mathbb{Z}$ by $\mathrm{K}_{j}(k)$. Since $\operatorname{rk}(1)=1$, the composition

$$
1 \otimes \mathrm{K}_{j}(k) \subset R(G) \otimes \mathrm{K}_{j}(k) \rightarrow \operatorname{Coker}\left(r_{j}\right)
$$

provides a splitting, and hence the inverse to this morphism.

Proof of Proposition 2.18. (1) By Proposition 2.5, $\mathrm{K}_{0}^{s g}\left(\mathbb{A}^{n} / G\right)$ is a torsion group annihilated by $|G|^{n-1}$, hence the same is true for $T_{j}$.

For even $j, \mathrm{~K}_{j}(k)$ of an algebraically closed field is torsion-free by a result of Suslin [67], hence $T_{j}=0$ for even $j$.

For odd $j$, and every $n \geq 1$, the $n$-torsion subgroup $\mathrm{K}_{j}(k)$ is finite [67], and since $\mathrm{K}_{0}^{s g}\left(\mathbb{A}^{n} / G\right)$ is a finite abelian group, $T_{j}$ is finite as well.

(2) The key in proving (2.4) is to compare the localization sequence for G-theory of $\mathbb{A}^{n} / G$ to the $G$-equivariant localization sequence for K-theory of $\mathbb{A}^{n}$. The two sequences are related by pushforward morphisms:

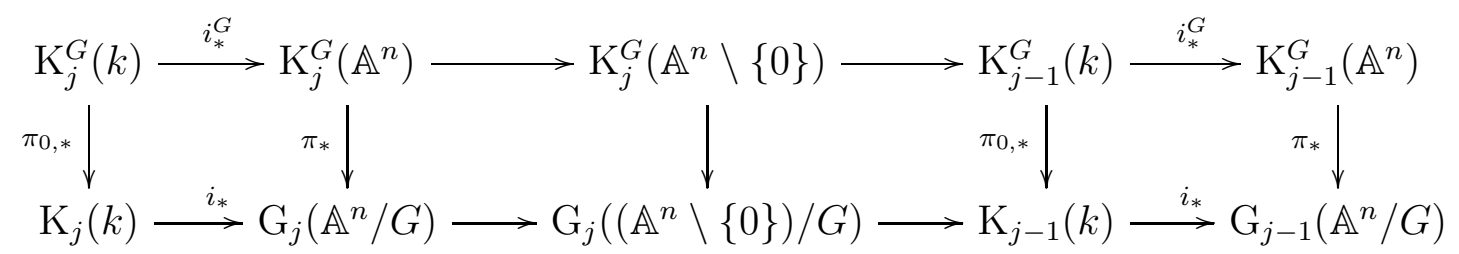

Here $i: \operatorname{Spec}(k) \rightarrow \mathbb{A}^{n} / G$ is the origin (unique fixed point of the action), and $\pi$ (resp. $\pi_{0}$ ) is the canonical morphism from the quotient Deligne-Mumford stack $\left[\mathbb{A}^{n} / G\right](\operatorname{resp} .[\operatorname{Spec}(k) / G])$ to its coarse moduli space, as in Lemma 2.22,

The morphisms $i_{*}: \mathrm{G}_{j}(k) \rightarrow \mathrm{G}_{j}\left(\mathbb{A}^{n} / G\right)$ are zero as they factor through the push-forward $\mathrm{G}_{j}(k) \rightarrow \mathrm{G}_{j}\left(\mathbb{A}^{n}\right)$ which are zero maps by the Bass formula in G-theory [58, chapter 6 Theorem 8. ii]. Thus the localization sequence for G-theory of $\mathbb{A}^{n} / G$ splits into short exact sequences. 
Using isomorphisms given by Lemma 2.22, from the commutative ladder (2.7) for every $j \geq 1$ we obtain the diagram:

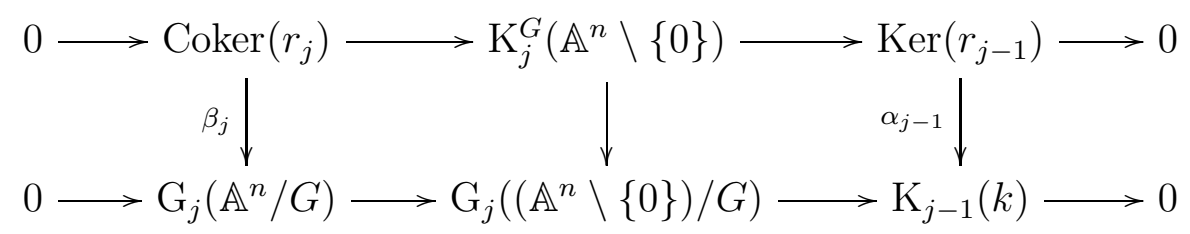

Since 0 is the only fixed point of the action, the action of $G$ on $\mathbb{A}^{n} \backslash\{0\}$ is free, so that the middle vertical map is an isomorphism and using the Snake Lemma we deduce an isomorphism

$$
\operatorname{Ker}\left(\alpha_{j-1}\right) \simeq \operatorname{Coker}\left(\beta_{j}\right) .
$$

From Lemma 2.22 (2) we get $\operatorname{Ker}\left(\alpha_{j}\right) \simeq T_{j}$ and from Lemma 2.22 (3) we get $\operatorname{Coker}\left(\beta_{j}\right) \simeq$ $\operatorname{Coker}\left(p^{*}: \mathrm{K}_{j}(k) \rightarrow \mathrm{G}_{j}\left(\mathbb{A}^{n} / G\right)\right)$. Since $p^{*}$ is injective (it is split by any smooth point $x_{1} \in$ $\left.\mathbb{A}^{n} / G\right)$, we obtain the exact sequence (2.4).

(3) Since $\mathbb{A}^{n} / G$ is contractible by Lemma 1.19, one gets (2.5) by plugging in (2.4) into the exact sequence of Proposition 1.20 (3).

\subsection{The global case.}

Theorem 2.23. Let $k$ be an algebraically closed field of characteristic zero and let $X$ be an $n$-dimensional quasi-projective variety. Assume that $X$ has only isolated quotient singularities $x_{1}, \ldots, x_{m}$ with isotropy groups $G_{1}, \ldots, G_{m}$, i.e. the completions $\widehat{\mathcal{O}}_{X, x_{i}}$ are isomorphic to $\widehat{\mathcal{O}}_{\mathbb{A}^{n} / G_{i}, 0}$ where each $G_{i} \subset \mathrm{GL}_{n}(k)$ is a finite group acting freely away from the origin. Then

(1) $\mathrm{K}_{0}^{s g}(X) \subset \mathbb{K}_{0}^{s g}(X)$ are finite abelian groups, annihilated by $\operatorname{lcm}\left(\left|G_{1}\right|, \ldots,\left|G_{m}\right|\right)^{n-1}$.

(2) $\mathrm{K}_{1}^{s g}(X)=0$.

(3) For all $j \geq 1$, we have $\mathrm{K}_{-j}^{s g}(X)=0$.

In addition, if $\operatorname{dim}(X)=2$, then $\mathbb{K}_{0}^{s g}(X) \simeq \widehat{G_{1}} \times \ldots \times \widehat{G_{m}}$.

Proof. By Orlov's Completion Theorem 1.5 and Corollary 2.3 we obtain equivalences

$$
\overline{\mathcal{D}^{\mathrm{sg}}(X)} \simeq \bigoplus_{i=1}^{m} \overline{\mathcal{D}^{\mathrm{sg}}\left(\mathbb{A}^{n} / G_{i}\right)} \simeq \bigoplus_{i=1}^{m} \mathcal{D}^{\mathrm{sg}}\left(\mathbb{A}^{n} / G_{i}\right) .
$$

induced by functors between dg-enhancements.

Thus by definition of the singularity K-theory groups and Remark 1.9 we have

$$
\mathrm{K}_{0}^{s g}(X) \subset \mathbb{K}_{0}^{s g}(X) \simeq \bigoplus_{i=1}^{m} \mathrm{~K}_{0}^{s g}\left(\mathbb{A}^{n} / G_{i}\right)
$$

and for $j \neq 0$

$$
\mathrm{K}_{j}^{s g}(X) \simeq \bigoplus_{i=1}^{m} \mathrm{~K}_{j}^{s g}\left(\mathbb{A}^{n} / G_{i}\right)
$$


Now (1) follows from Propositions 2.5, 2.9, (2) follows from Proposition 2.19 and (3) follows from Proposition 2.1.

Finally if $\operatorname{dim}(X)=2$, we have isomorphisms $\mathrm{K}_{0}^{s g}\left(\mathbb{A}^{n} / G_{i}\right)=\widehat{G_{i}}$ by Proposition $2.8\left(G_{i}\right.$ acts freely on $\mathbb{A}^{n} \backslash\{0\}$ and in particular has no reflections) so that in this case $\mathbb{K}_{0}^{s g}(X) \simeq \widehat{G_{1}} \times \cdots \times \widehat{G_{m}}$.

Corollary 2.24. Under the assumptions of Theorem 2.23 the following is true.

(1) We have an exact sequence $0 \rightarrow \mathrm{K}_{0}(X) \rightarrow \mathrm{G}_{0}(X) \rightarrow \mathrm{K}_{0}^{s g}(X) \rightarrow 0$.

$(2) \mathrm{K}_{-1}(X)$ is a finite torsion abelian group satisfying the same condition on orders as $\mathrm{K}_{0}^{s g}(X)$ (see Theorem $\left.2.23(1)\right)$.

(3) For all $j \geq 2$, we have $\mathrm{K}_{-j}(X)=0$.

Proof. This follows from Theorem 2.23 and Lemma 1.10.

Remark 2.25. The injectiviy of the canonical map $\mathrm{K}_{0}(X) \rightarrow \mathrm{G}_{0}(X)$ will generally fail if either:

(a) X has quotient singularities which are not isolated, see Example 3.7

(b) $X$ has an isolated rational singularity which is not a quotient singularity, see Example 3.8

Remark 2.26. We do not know if $\mathrm{K}_{0}^{s g}(X)=\operatorname{Coker}\left(\mathrm{K}_{0}(X) \rightarrow \mathrm{G}_{0}(X)\right)$ is torsion for any variety $X$ with quotient singularities, not necessarily isolated ones. The result is known to be true for simplicial toric varieties [14].

Example 2.27. One of the simplest examples of a projective surface $X$ with quotient singularities and non-vanishing $\mathrm{K}_{-1}(X)$ is the following one. Consider $G=\mathbb{Z}_{2}$ acting on $\mathbb{P}^{1}$ via $[x: y] \mapsto[x:-y]$ and let $X=\left(\mathbb{P}^{1} \times \mathbb{P}^{1}\right) / \mathbb{Z}_{2}$ where the action is diagonal. Thus $X$ has four ordinary double points as singularities. Using [76] one can compute that $\mathrm{K}_{-1}(X)=\mathbb{Z}_{2}$.

2.4. Relation to the resolution of singularities. Let $\pi: Y \rightarrow X$ be a resolution of singularities. Here $X$ is a variety and $Y$ is a variety or more generally a Deligne-Mumford stack. If we assume that singularities of $X$ are rational which by definition means that $\pi_{*} \mathcal{O}_{Y}=\mathcal{O}_{X}$, using the projection formula we get a commutative diagram

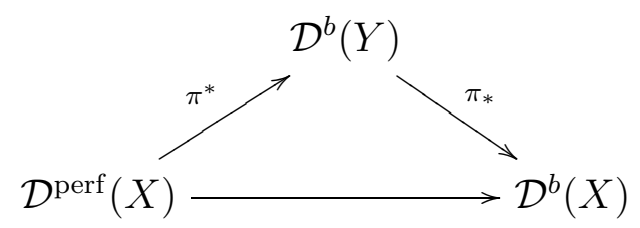

where the functors $\mathcal{D}^{\text {perf }}(X) \rightarrow \mathcal{D}^{b}(X)$ and $\pi^{*}$ are both fully faithful. 
We also get an induced diagram on the Grothendieck groups

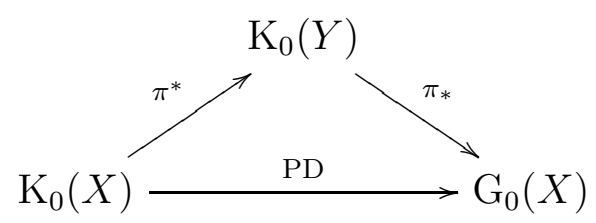

Theorem 2.28. If $X$ is a quasi-projective variety over an algebraically closed field of characteristic zero and with only isolated quotient singularities, then $\pi^{*}: \mathrm{K}_{0}(X) \rightarrow \mathrm{K}_{0}(Y)$ is injective. Proof. By Corollary 2.24, $\mathrm{K}_{0}(X) \stackrel{\mathrm{PD}}{\rightarrow} \mathrm{G}_{0}(X)$ is injective. Injectivity of $\pi^{*}$ follows from the diagram (2.8).

Remark 2.29. The injectivity of $\pi^{*}: \mathrm{K}_{0}(X) \rightarrow \mathrm{K}_{0}(Y)$ does not follow from the fact that $\pi^{*}: \mathcal{D}^{\text {perf }}(X) \rightarrow \mathcal{D}^{b}(Y)$ is fully faithful and will generally fail for rational singularities. Indeed in Examples 3.7, 3.8 varieties with rational singularities have huge $\mathrm{K}_{0}(X)$, but admit resolutions with finitely generated $\mathrm{K}_{0}(Y)$.

In dimension up to three, Theorem [2.28 has been known since the work of Levine [46, Corollary 3.4] and for normal surfaces with rational singularities an analogous result follows from the work of Krishna and Srinivas [44, Corollary 1.5].

There is an apparent duality between $\pi_{*}$ and $\pi^{*}$ in the diagram (2.8). Instead of injectivity of $\pi^{*}$ we can ask about surjectivity of $\pi_{*}$, which indeed sometimes holds.

Theorem 2.30. Let $X$ be a variety over a field $k$ characteristic zero with quotient singularities (not necessarily isolated) and let $\pi: Y \rightarrow X$ be a resolution of singularities, where $Y$ is a variety or more generally a Deligne-Mumford stack. Then:

(1) The pushforward $\pi_{*}: \mathcal{D}^{b}(Y) \rightarrow \mathcal{D}^{b}(X)$ is essentially surjective.

(2) The pushforward induces an exact equivalence

$$
\mathcal{D}^{b}(Y) / \operatorname{ker}\left(\pi_{*}\right) \stackrel{\simeq}{\rightarrow} \mathcal{D}^{b}(X) .
$$

In particular $\pi_{*}: \mathrm{K}_{0}(Y) \rightarrow \mathrm{G}_{0}(X)$ is surjective.

Lemma 2.31. If $k$ is a field of characteristic zero and the statement (1) or (2) of Theorem 2.30 holds for a single resolution $\pi: Y \rightarrow X$, then it holds for all resolutions of $X$.

Proof. The proof is a standard application of the Weak Factorization Theorem [77, 1], extended to Deligne-Mumford stacks in [8]. Given a birational isomorphism between Deligne-Mumford orbifolds, that is Deligne-Mumford stacks with trivial generic stabilizers, it can be decomposed into a sequence of stacky blows ups and blow downs with smooth centers. This means that given two resolutions $\pi: Y \rightarrow X, \pi^{\prime}: Y^{\prime} \rightarrow X$ we may assume that $Y^{\prime}$ is obtained from $Y$ by a single smooth stacky blow up $\gamma: Y^{\prime} \rightarrow Y$. Recall that by definition a stacky blow up is 
either a blow up of a substack, or a root stack along a smooth divisor, and in each case we have $\gamma_{*} \mathcal{O}_{Y^{\prime}} \simeq \mathcal{O}_{Y}$ (see e.g. [9, Example 4.6]).

We get a commutative diagram

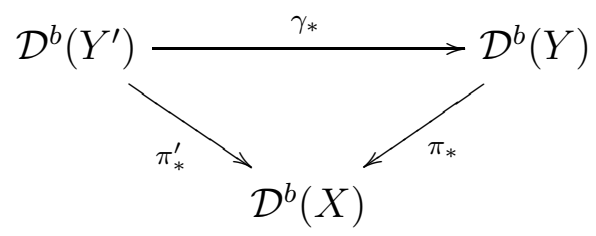

Furthermore the adjoint pair $\gamma^{*}, \gamma_{*}$ satisfies $\gamma_{*} \gamma^{*}=$ id so that $\gamma^{*}$ is fully-faithful and there is a semi-orthogonal decomposition

$$
\mathcal{D}^{b}\left(Y^{\prime}\right)=\left\langle\operatorname{Ker}\left(\gamma_{*}\right), \gamma^{*} \mathcal{D}^{b}(Y)\right\rangle
$$

In particular $\gamma_{*}$ is essentially surjective and condition (1) for $Y$ is equivalent to condition (1) for $Y^{\prime}$. Furthermore we have a semi-orthogonal decomposition

$$
\operatorname{Ker}\left(\pi_{*}^{\prime}\right)=\left\langle\operatorname{Ker}\left(\gamma_{*}\right), \gamma^{*} \operatorname{Ker}\left(\pi_{*}\right)\right\rangle
$$

which induces an equivalence of Verdier localizations

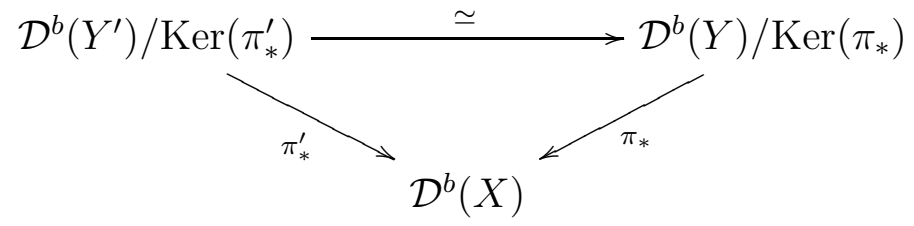

so that conditions (2) for $Y$ and $Y^{\prime}$ are equivalent as well.

Lemma 2.32. If $\pi: Y \rightarrow X$ is a resolution of rational singularities and $\mathcal{D}^{-}(Y)$ admits a $t$-structure which induces a bounded $t$-structure on $\mathcal{D}^{b}(Y)$ and for which $\pi_{*}: \mathcal{D}^{-}(Y) \rightarrow \mathcal{D}^{-}(X)$ is $t$-exact, then (1) and (2) of Theorem 2.30 are true.

Proof. We temporarily use the notation $\operatorname{Ker}^{b}\left(\pi_{*}\right):=\operatorname{Ker}\left(\pi_{*}\right) \cap \mathcal{D}^{b}(Y)$. We will show that the functor $\overline{\pi_{*}}: \mathcal{D}^{b}(Y) / \operatorname{Ker}^{b}\left(\pi_{*}\right) \rightarrow \mathcal{D}^{b}(X)$ is essentially surjective and fully faithful.

Essential surjectivity is proved in the same way as in [43, Corollary 2.5]. For every $\mathcal{E} \in \mathcal{D}^{b}(X)$ and $N \geq 1$ we consider the distinguished triangle

$$
\pi^{*} \mathcal{E} \rightarrow \tau_{\mathcal{A}}^{\geq-N} \pi^{*} \mathcal{E} \rightarrow C,
$$

where $\tau_{\mathcal{A}}^{\geq-N}$ denotes the truncation with respect to the corresponding $t$-structure $\mathcal{A}$ on $\mathcal{D}^{-}(Y)$. We apply $\pi_{*}$ to this triangle. Since $\pi_{*} \pi^{*}=$ id and $\pi_{*}$ is $t$-exact, in particular $\pi_{*}$ commutes with truncation functors, the push-forward of the triangle above has the form:

$$
\mathcal{E} \rightarrow \tau^{\geq-N} \mathcal{E} \rightarrow \pi_{*} C,
$$


where $\tau^{\geq-N}$ is the truncation with respect to the standard $t$-structure on $\mathcal{D}^{-}(X)$. Since $\mathcal{E}$ is bounded, for sufficiently large $N$ we have $\pi_{*} C=0$ so that $C \in \operatorname{Ker}\left(\pi_{*}\right)$. In particular, $\overline{\pi_{*}}: \mathcal{D}^{b}(Y) / \operatorname{Ker}^{b}\left(\pi_{*}\right) \rightarrow \mathcal{D}^{b}(X)$ is essentially surjective.

On the other hand, one observes by the diagram

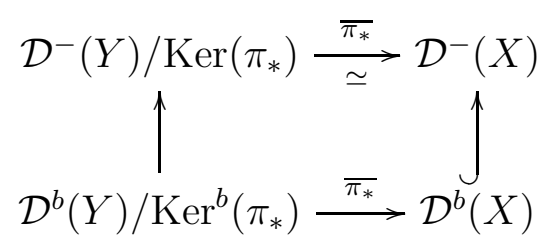

that $\overline{\pi_{*}}$ is fully faithful if and only if the natural functor

$$
\mathcal{D}^{b}(Y) / \operatorname{Ker}^{b}\left(\pi_{*}\right) \rightarrow \mathcal{D}^{-}(Y) / \operatorname{Ker}\left(\pi_{*}\right)
$$

is fully faithful. To show this, we use Verdier's criterion [74, Theorem 2.4.2]. We see that if $C \rightarrow B$ is a morphism in $\mathcal{D}^{-}(Y)$ with $C \in \operatorname{Ker}\left(\pi_{*}\right)$ and $B \in \mathcal{D}^{b}(Y)$, then for a big enough $N$ (depending on $B$ ) this morphism factors through $\tau_{\overline{\mathcal{A}}}^{\geq-N} C$. Since $\pi_{*}$ commutes with $\tau_{\mathcal{A}}^{\geq-N}$, one easily sees that $\tau_{\mathcal{A}}^{\geq-N} C \in \operatorname{Ker}^{b}\left(\pi_{*}\right)$.

Proof of Theorem 2.30. By Lemma 2.31 it suffices to check the statement for a single resolution. We consider the canonical stack $\pi: \mathcal{X}_{\text {can }} \rightarrow X$ over $X$ [27, Remark 4.9]. Since we assume $k$ has characteristic zero, the pushforward $\pi_{*}: \mathcal{X}_{c a n} \rightarrow X$ is exact. The proof is finished using Lemma 2.32.

Remark 2.33. Statements (1) and (2) of Theorem 2.30 for a resolution of arbitrary rational singularities $\pi: Y \rightarrow X$ is an old open question going back to Bondal and Orlov [12]. In addition to quotient singularities the answer is positive in the case of cones over smooth Fano varieties [26], and for rational singularities such that fibers of a resolution $Y \rightarrow X$ have dimension at most one [43] (in [43, Corollary 2.5] property (1) is proved, while property (2) follows from Lemma 2.32).

\section{Examples and Applications}

In this section $k$ is an algebraically closed field of characteristic zero.

\subsection{Torsion-free $\mathrm{K}_{0}(X)$.}

Application 3.1 ([45, 46]). Let $X$ be a projective rational surface with isolated quotient singularities. Then $\mathrm{K}_{0}(X)$ is a free abelian group of the same rank as $\mathrm{G}_{0}(X)$.

Indeed if $\pi: Y \rightarrow X$ is a resolution, then by Theorem 2.28 we have an injection $\pi^{*}$ : $\mathrm{K}_{0}(X) \rightarrow \mathrm{K}_{0}(Y)$. Since $Y$ is a smooth projective rational surface, $\mathrm{K}_{0}(Y)$ is a free abelian group of finite rank, and the same is true for $\mathrm{K}_{0}(X)$. Finally by Corollary 2.24, we have an inclusion $\mathrm{K}_{0}(X) \subset \mathrm{G}_{0}(X)$ and the ranks of the two groups are equal.

Note that $\mathrm{G}_{0}(X)$ will typically have non-zero torsion. 
Application 3.2 (Weighted projective spaces with coprime weights). Let $X=\mathbb{P}\left(a_{0}, \ldots, a_{n}\right)$ be a weighted projective space. Let us assume that the weights $a_{0}, \ldots, a_{n}$ are pairwise coprime. In this case singularities of $X$ are isolated, and using our results we show that $\mathrm{K}_{0}(X)$ is a free abelian group of rank $n+1$.

Indeed if we let $Y=\left[\mathbb{P}\left(a_{0}, \ldots, a_{n}\right)\right]$ to be the weighted projective stack, the natural morphism $\pi: Y \rightarrow X$ is a resolution of singularities, and by Theorem 2.28, $\pi^{*}$ is injective. Since $\mathrm{K}_{0}(Y)$ is a free abelian of finite rank [42, Theorem 5.6], the same is true for $\mathrm{K}_{0}(X)$. To compute the rank of $\mathrm{K}_{0}(X)$ we can use the following argument: by Corollary 2.24 we have an isomorphism $\mathrm{K}_{0}(X) \otimes \mathbb{Q} \simeq \mathrm{G}_{0}(X) \otimes \mathbb{Q}$ and the latter space is $(n+1)$-dimensional, which can be seen by comparing $\mathrm{G}_{0}(X)$ to Chow groups $\left[2\right.$. Thus we get $\mathrm{K}_{0}(X) \simeq \mathbb{Z}^{n+1}$.

3.2. $A D E$ curves and threefolds. We consider one-dimensional $A D E$ singularities over an algebraically closed field of characteristic zero. For each such curve $C$ we compute $\operatorname{Pic}(C)$ as well as $\mathrm{K}_{0}^{s g}(C)$ and $\mathrm{K}_{1}^{s g}(C)$. If $N$ is the number of irreducible components of the curve, then $\mathrm{K}_{0}^{s g}(C)$ is computed using Corollary 1.28, We record the results in the table:

\begin{tabular}{|c|c|c|c|c|c|}
\hline$C$ & Equation & $N$ & $\mathrm{~K}_{0}^{s g}(C)$ & $\operatorname{Pic}(C)$ & $\mathrm{K}_{1}^{s g}(C)$ \\
\hline$A_{2 l}, l \geq 1$ & $y^{2}+z^{2 l+1}$ & 1 & 0 & $k^{l}$ & $k^{l}$ \\
\hline$A_{2 l-1}, l \geq 1$ & $y^{2}+z^{2 l}$ & 2 & $\mathbb{Z}$ & 0 & $k^{*} \oplus \mathbb{Z}$ \\
\hline$D_{2 l}, l \geq 2$ & $y^{2} z+z^{2 l-1}$ & 3 & $\mathbb{Z}^{2}$ & 0 & $\left(k^{*} \oplus \mathbb{Z}\right)^{2}$ \\
\hline$D_{2 l-1}, l \geq 3$ & $y^{2} z+z^{2 l-2}$ & 2 & $\mathbb{Z}$ & $k^{l-2}$ & {$\left[k^{*} \oplus \mathbb{Z} ; k^{l-2}\right]$} \\
\hline$E_{6}$ & $y^{3}+z^{4}$ & 1 & 0 & $k^{3}$ & $k^{3}$ \\
\hline$E_{7}$ & $y^{3}+y z^{3}$ & 2 & $\mathbb{Z}$ & $k$ & {$\left[k^{*} \oplus \mathbb{Z} ; k\right]$} \\
\hline$E_{8}$ & $y^{3}+z^{5}$ & 1 & 0 & $k^{4}$ & $k^{4}$ \\
\hline
\end{tabular}

We use the notation $[A ; B]$ to denote an abelian group which has a subgroup $A$ with quotient $B$. The first singularity K-theory groups $\mathrm{K}_{1}^{s g}(C)$ are computed using the following Proposition.

Proposition 3.3. For every ADE singularity we have a natural exact sequence

$$
0 \rightarrow\left(k^{*} \oplus \mathbb{Z}\right)^{N-1} \rightarrow \mathrm{K}_{1}^{s g}(C) \rightarrow \operatorname{Pic}(C) \rightarrow 0 .
$$

Proof. By Proposition 1.20 (3) and using the fact that $\mathrm{K}_{0}(C)=\mathbb{Z} \oplus \operatorname{Pic}(C)$ (see [32, Remark 1 on page 126]) we get a short exact sequence

$$
0 \rightarrow \mathrm{G}_{1}(C) / k^{*} \rightarrow \mathrm{K}_{1}^{s g}(C) \rightarrow \operatorname{Pic}(C) \rightarrow 0 .
$$

To finish the proof we show that $\mathrm{G}_{1}(C)=\left(k^{*}\right)^{N} \oplus \mathbb{Z}^{N-1}$, and the morphism $\mathrm{K}_{1}(k) \rightarrow \mathrm{G}_{1}(C)$ maps $k^{*}$ into $\left(k^{*}\right)^{N}$ diagonally. This is done using the localization sequence for the closed embedding $i:\{0\} \rightarrow C$. Since for every $j \geq 0, i_{*}: \mathrm{G}_{j}(k) \rightarrow \mathrm{G}_{j}(C)$ factors through any 
component $\mathbb{A}^{1}$ of the normalization of $C$, it is a zero map, and we get a short exact sequence

$$
0 \rightarrow \mathrm{G}_{1}(C) \rightarrow \mathrm{G}_{1}\left(\mathbb{A}^{1} \backslash\{0\}\right)^{N} \rightarrow \mathrm{G}_{0}(k) \rightarrow 0
$$

which finishes the proof as $\mathrm{G}_{1}\left(\mathbb{A}^{1} \backslash\{0\}\right)=k^{*} \oplus \mathbb{Z}$.

Lemma 3.4. If $C$ is a curve with equation $x^{a}-y^{b}=0$ where $\operatorname{gcd}(a, b)=1$, then we have an isomorphism

$$
\operatorname{Pic}(C) \simeq k^{\frac{1}{2}(a-1)(b-1)} .
$$

Proof. Consider $\pi: \mathbb{A}^{1} \rightarrow C$ given by $\pi(t)=\left(t^{b}, t^{a}\right)$. Under the condition $\operatorname{gcd}(a, b)=1$, $\pi$ is surjective which implies irreducibility of $C$, and since $\pi$ is finite of degree one, $\pi$ is the normalization morphism. By [54, Corollary 3.3] we get an isomorphism of abelian groups

$$
\operatorname{Pic}(C) \simeq k[t] / k\left[t^{a}, t^{b}\right]
$$

Thus $\operatorname{Pic}(C)$ obtains a $k$-vector space structure with a $k$-basis corresponding of $t^{i}$, for every $i \geq 0$ which can not be represented as a non-negative integer combination of $a$ and $b$.

By a classical theorem of Sylvester, the number of positive integers not representable by non-negative integer combinations of $a$ and $b$ is equal to $\frac{1}{2}(a-1)(b-1)$ (see [53] for a modern treatment) so that we have an isomorphism of abelian groups

$$
k[t] / k\left[t^{a}, t^{b}\right] \simeq k^{\frac{1}{2}(a-1)(b-1)} .
$$

Every $A D E$ curve $C$ is a union of components isomorphic to $\mathbb{A}^{1}$ and at most one component $C_{0}$ with equation $x^{a}-y^{b}=0$. Trivializing line bundles on each affine line component, we deduce that $\operatorname{Pic}(C)=\operatorname{Pic}\left(C_{0}\right)$. Proposition 3.3 and Lemma 3.4 allow us to fill in the table (3.1).

We demonstrate what the singularity K-theory has to do with the question of computing class groups. The applications below can be obtained by other methods too, however, we demonstrate the approach which relies on Knörrer periodicity shifting the topological filtration (Proposition 1.30).

Application 3.5. Let $C \subset \mathbb{A}^{2}$ with coordinates $z, w$ be given by $g(z, w)=0$ and let $X \subset \mathbb{A}^{4}$ with coordinates $x, y, z, w$ be given by $x y+g(z, w)=0$.

Let us assume that $C$ is reduced. Since we have $\operatorname{Sing}(X)=\{(0,0)\} \times \operatorname{Sing}(C)$, the latter condition is equivalent to $X$ having isolated singularities, and since $X$ is a hypersurface, it is irreducible and normal.

Let $N$ be the number of irreducible components of $C$. By Proposition 1.30 and Proposition 1.24 we have an isomorphism

$$
\mathrm{Cl}(X) / \operatorname{Pic}(X)=\operatorname{gr}^{1} \mathrm{~K}_{0}^{s g}(X) \simeq \operatorname{gr}^{0} \mathrm{~K}_{0}^{s g}(C)=\mathbb{Z}^{N-1},
$$

in particular $X$ is factorial if and only if $C$ is irreducible. 
Example 3.6. We can compute the class group of the standard forms of three-dimensional ADE singularities. Since $X$ is given by a weighted homogeneous equation, $\operatorname{Pic}(X)=0$ [51, Lemma 5.1] we have $\mathrm{Cl}(X) \simeq \mathrm{K}_{0}^{s g}(X)=\mathbb{Z}^{N-1}$. We put the results in the table (cf. table [3.1):

\begin{tabular}{|c|c|c|}
\hline$X$ & Equation & $\mathrm{Cl}(X)$ \\
\hline$A_{2 k}(k \geq 1)$ & $x y+z^{2}+w^{2 k+1}$ & 0 \\
\hline$A_{2 k-1}(k \geq 1)$ & $x y+z^{2}+w^{2 k}$ & $\mathbb{Z}$ \\
\hline$D_{2 k}(k \geq 2)$ & $x y+z^{2} w+w^{2 k-1}$ & $\mathbb{Z}^{2}$ \\
\hline$D_{2 k-1}(k \geq 3)$ & $x y+z^{2} w+w^{2 k-2}$ & $\mathbb{Z}$ \\
\hline$E_{6}$ & $x y+z^{3}+w^{4}$ & 0 \\
\hline$E_{7}$ & $x y+z^{3}+z w^{3}$ & $\mathbb{Z}$ \\
\hline$E_{8}$ & $x y+z^{3}+w^{5}$ & 0 \\
\hline
\end{tabular}

3.3. Non-vanishing $\mathrm{K}_{1}^{s g}(X)$. In this section we collect some examples where $\mathrm{K}_{1}^{s g}(X)$ is nonzero. From the singularity K-theory exact sequence (1.1) it follows that $\mathrm{K}_{1}^{s g}(X)$ surjects onto $\operatorname{Ker}\left(\mathrm{K}_{0}(X) \stackrel{\mathrm{PD}}{\rightarrow}\right.$ $\left.\mathrm{G}_{0}(X)\right)$.

Example 3.7 (Non-isolated quotient singularity with huge kernel $\operatorname{Ker}\left(\mathrm{K}_{0}(X) \rightarrow \mathrm{G}_{0}(X)\right)$. The first such example has been constructed by Gubeladze [38. We present an example given by Cortiñas, Haesemeyer, Walker and Weibel [18, Example 5.10].

Let $E=\mathcal{O} \oplus \mathcal{O}(2)$ be the rank two bundle over $\mathbb{P}^{1}$. Let $\mathbb{Z}_{2}$ act on $E$ fiberwise via $v \mapsto-v$. Then $X=E / \mathbb{Z}_{2}$, has quotient singularities and its singular locus isomorphic to $\mathbb{P}^{1}$.

The canonical map $\mathrm{K}_{0}(X) \rightarrow \mathrm{G}_{0}(X)$ is not injective, and furthermore, the kernel $\operatorname{Ker}\left(\mathrm{K}_{0}(X) \rightarrow\right.$ $\left.\mathrm{G}_{0}(X)\right)$ is huge, that is contains the base field $k$ as a subgroup.

Example 3.8 (Isolated rational singularity with huge kernel $\operatorname{Ker}\left(\mathrm{K}_{0}(X) \rightarrow \mathrm{G}_{0}(X)\right)$. Consider a smooth cubic hypersurface $S \subset \mathbb{P}^{3}$, and let $X \subset \mathbb{A}^{4}$ be the affine cone over $S$. Then $X$ has an isolated rational singularity.

The Grothendieck group of a cone over a smooth variety has been computed in [20]. In particular since $\chi\left(\mathcal{T}_{S}\right)=-4$ by Riemann-Roch so that $H^{1}\left(S, \Omega_{S / \mathbb{Q}}^{1}(1)\right)=H^{1}\left(S, \Omega_{S / k}^{1}(1)\right)=$ $H^{1}\left(S, \mathcal{T}_{S}\right) \neq 0$, where the first equation follows from the short exact sequence

$$
0 \rightarrow \Omega_{k / \mathbb{Q}}^{1}(1) \rightarrow \Omega_{S / \mathbb{Q}}^{1}(1) \rightarrow \Omega_{S / k}^{1}(1) \rightarrow 0,
$$

see [36, Proposition 20.6.2]. The main result of [20] implies that $\mathrm{K}_{0}(X)$ is huge, that is it contains a nonzero $k$-vector space.

Finally by Proposition 1.20 (1) the canonical map $\mathrm{K}_{0}(X) \rightarrow \mathrm{G}_{0}(X)$ factors through $\mathbb{Z}$, so that $\operatorname{Ker}\left(\mathrm{K}_{0}(X) \rightarrow \mathrm{G}_{0}(X)\right)$ is huge as well.

Example 3.9 (Non-vanishing $\mathrm{K}_{1}^{s g}(X)$ for isolated quotient singularities over non-algebraically closed fields). Let $X=\mathbb{A}^{2} / \mathbb{Z}_{2}$ be the quotient by the action $v \mapsto-v$. We claim that $\mathrm{K}_{1}^{\text {sg }}(X) \simeq$ $k^{*} /\left(k^{*}\right)^{2}$. 
Indeed, $X$ is isomorphic to the affine surface $x y+z^{2}=0$, and using the Knörrer periodicity Theorem 1.3 we have

$$
\mathrm{K}_{1}^{s g}(X) \simeq \mathrm{K}_{1}^{s g}(R),
$$

where $R=k[\epsilon] /\left(\epsilon^{2}\right)$. We compute the singularity $\mathrm{K}$-theory via the $\mathrm{K}$-theory sequence (1.1), plugging in $\mathrm{G}_{i}(R)=\mathrm{K}_{i}(k)$, as $\mathrm{G}$-theory is independent of the non-reduced scheme structure:

$$
\mathrm{K}_{1}(R) \rightarrow k^{*} \rightarrow \mathrm{K}_{1}^{s g}(R) \rightarrow \mathrm{K}_{0}(R) \rightarrow \mathbb{Z} \rightarrow \mathrm{K}_{0}^{s g}(R) \rightarrow 0 .
$$

Now $\mathrm{K}_{0}(R)=\mathbb{Z}$ and the map $\mathbb{Z}=\mathrm{K}_{0}(R) \rightarrow \mathbb{Z}$ is multiplication by two (cf Example 1.21), and similarly $\mathrm{K}_{1}(R)=R^{*}$, and the map $\mathrm{K}_{1}(R) \rightarrow k^{*}$ is $a+b \epsilon \mapsto a^{2}$ [41, Example 10.2]. We get

$$
\mathrm{K}_{1}^{s g}(X) \simeq \mathrm{K}_{1}^{s g}(R) \simeq k^{*} /\left(k^{*}\right)^{2}
$$

which is in general a non-finitely generated 2-torsion group.

3.4. Proof of a conjecture of Srinivas for quotient singularities. In the 1980s Srinivas considered the question whether for an isolated quotient singularity $x_{0} \in X$ the length homomorphism $l: \mathrm{K}_{0}\left(X\right.$ on $\left.x_{0}\right) \rightarrow \mathbb{Z}$ is an isomorphism [64, Page 38]. Here $\mathrm{K}_{0}\left(X\right.$ on $\left.x_{0}\right)$ stands for the Grothendieck group of perfect complexes supported at $x_{0}$ (originally Srinivas has considered the Grothendieck group of coherent sheaves which are supported at the singular points and which are perfect as complexes, but by [59, Proposition 2] these two groups are isomorphic).

Levine has proved that $l$ is an isomorphism if $X$ is two-dimensional with isolated quotient singularities [45, Theorem 3.2], that $l$ is always surjective for isolated Cohen-Macaulay singularities, and that it has torsion kernel [46, Proposition 2.6, Theorem 2.7] in the case of isolated quotient-singularities.

The language of the singularity K-theory is well-adapted to deal with this kind of questions.

Lemma 3.10. Let $k$ be an algebraically closed field. Let $X$ be a quasi-projective variety with isolated singularities. There is an exact sequence

$$
\mathrm{K}_{1}^{s g}(X) \rightarrow \mathrm{K}_{0}(X \text { on } \operatorname{Sing}(X)) \stackrel{l}{\rightarrow} \mathbb{Z}^{\operatorname{Sing}(X)} \rightarrow 0
$$

and a natural surjective homomorphism $\operatorname{Ker}(l) \rightarrow \operatorname{Ker}\left(\mathrm{PD}: \mathrm{K}_{0}(X) \rightarrow \mathrm{G}_{0}(X)\right)$.

Proof. We consider the diagram of pretriangulated dg-categories

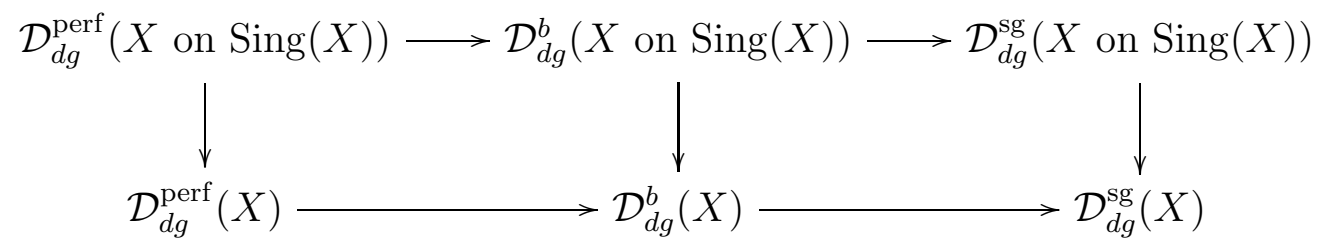


and the associated long exact sequences of Schlichting's K-groups:

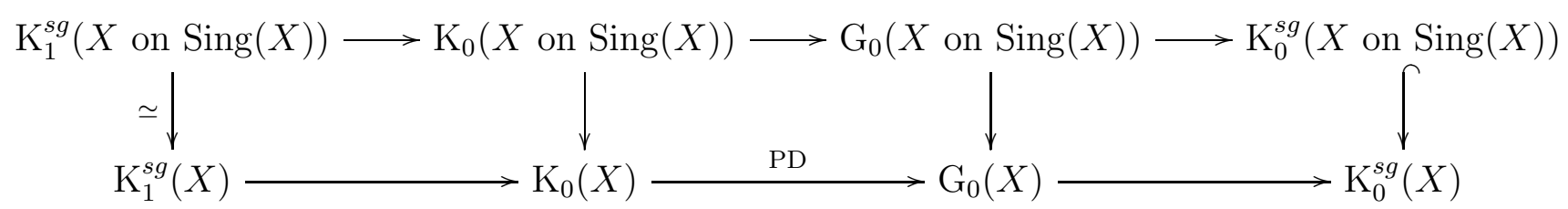

where the left vertical arrow is an isomorphism and the right vertical arrow is injective by Lemma 1.12.

We have $\mathrm{K}_{0}^{s g}(X$ on $\operatorname{Sing}(X))=0$ (Corollary 1.27), and we have a natural isomorphism

$$
\mathrm{G}_{0}(X \text { on } \operatorname{Sing}(X))=\mathrm{G}_{0}(\operatorname{Sing}(X))=\mathbb{Z}^{\operatorname{Sing}(X)}
$$

given by the length (dimension) of zero-dimensional coherent sheaves, so that exact sequence (3.3) is the first row in the diagram above.

Finally, the diagram above also induces the surjection $\operatorname{Ker}(l) \rightarrow \operatorname{Ker}(\mathrm{PD})$.

The next result deals with the injectivity part of the Srinivas conjecture for quotient singularities, and thus gives a stronger version of [45, Theorem 3.2].

Proposition 3.11. If $X$ is a quasi-projective variety with isolated quotient singularities then the length map

$$
l: \mathrm{K}_{0}(X \text { on } \operatorname{Sing}(X)) \rightarrow \mathbb{Z}^{\operatorname{Sing}(X)}
$$

is an isomorphism.

Proof. By Theorem $2.23(2), \mathrm{K}_{1}^{s g}(X)=0$. Lemma 3.10 implies the result.

Remark 3.12. By Lemma 3.10 non-vanishing of $\operatorname{Ker}\left(\mathrm{PD}: \mathrm{K}_{0}(X) \rightarrow \mathrm{G}_{0}(X)\right)$ implies nonvanishing of $\operatorname{Ker}(l)$. This applies for instance in the case of a cone over a smooth cubic surface, see Example 3.8 .

Example 3.13. Let $k$ be an arbitrary field with $\operatorname{char}(k) \neq 2$. Let $Q_{n}$ be the $n$-dimensional affine split quadric cone as in Examples 1.31, 1.32. It is a result of Levine [46, Theorem 4.2] that

$$
\mathrm{K}_{0}\left(Q_{n} \text { on } 0\right) \simeq \begin{cases}\mathbb{Z} \oplus k^{*} /\left(k^{*}\right)^{2}, & n \text { even } \\ \mathbb{Z}^{2} \oplus k^{*}, & n \text { odd }\end{cases}
$$

This result can be reproved using exact sequence (3.3) and the fact that

$$
\mathrm{K}_{1}^{s g}\left(Q_{n}\right) \simeq \begin{cases}k^{*} /\left(k^{*}\right)^{2}, & n \text { even }(\text { cf Example 3.9) } \\ \mathbb{Z} \oplus k^{*}, & n \text { odd }\left(\text { cf } A_{1} \text { case in (3.1) }\right)\end{cases}
$$

Similarly, one can compute $K_{0}(X$ on 0$)$ for other $A D E$ singularities of arbitrary dimension. We omit the details of this computation. 


\section{REFERENCES}

[1] D. Abramovich, K. Karu, K. Matsuki and J. Włodarczyk: Torification and factorization of birational maps, J. Amer. Math. Soc. 15(3):531-572, 2002.

[2] A. Al Amrani: Groupe de Chow et K-théorie cohérente des espaces projectifs tordus, Proceedings of Research Symposium on K-Theory and its Applications (Ibadan, 1987). K-Theory 2 (1989), no. 5, 579-602.

[3] M. Auslander and I. Reiten: Grothendieck group of algebras and orders, J. Pure Appl. Algebra 39 (1986), $1-51$.

[4] P. Balmer and M. Schlichting: Idempotent Completion of Triangulated Categories, J. of Algebra, 236 (2001), 819-834.

[5] P.Baum, W. Fulton and R. MacPherson: Riemann-Roch for singular varieties Publications Mathématiques de l'IHÉS 45 (1975), 101-145.

[6] A. Beilinson, V. Vologodsky: A DG guide to Voevodsky's motives, Geom. Funct. Anal. 17 (2008), no. 6, 1709-1787.

[7] D. J. Benson: Polynomial Invariants of Finite Groups, London Mathematical Society Lecture Note Series, 190. Cambridge University Press, Cambridge, 1993.

[8] D. Bergh: Functorial destackification of tame stacks with abelian stabilisers, Compositio Math. 153 (2017), $1257-1315$.

[9] D. Bergh, V. A. Lunts and O. M. Schnürer: Geometricity for derived categories of algebraic stacks Selecta Math. (N.S.) 22 (2016), no. 4, 2535-2568.

[10] D. Bergh, O.M. Schnürer: Conservative descent for semi-orthogonal decompositions, arXiv:1712.06845.

[11] P. Berthelot, A. Grothendieck, L. Illusie et al.: Théorie des intersections et Théorème de Riemann-Roch, Springer Lecture Notes in Mathematics, 225 (1971).

[12] A. Bondal, D. Orlov: Derived categories of coherent sheaves, Proceedings of the International Congress of Mathematicians, Vol. II (Beijing, 2002), 47-56, Higher Ed. Press, Beijing, 2002.

[13] R.O. Buchweitz: Maximal Cohen-Macaulay modules and Tate cohomology over Gorenstein rings, preprint, Univ. Hannover, 1986.

[14] M.Brion, M. Vergne: An equivariant Riemann-Roch theorem for complete, simplicial toric varieties, J. Reine Angew. Math. 482 (1997), 67-92.

[15] A. Canonaco and P. Stellari: Uniqueness of dg enhancements for the derived category of a Grothendieck category, arXiv: 1507.05509.

[16] C. Chevalley: Anneaux de Chow et Applications, Séminaire Chevalley, Sécretariat Math., Paris (1958).

[17] G. Cortiñas, C. Haesemeyer, M. Schlichting, C. Weibel: Cyclic homology, cdh-cohomology and negative Ktheory, Ann. of Math. (2) 167 (2008), no. 2, 549-573.

[18] G. Cortiñas, C. Haesemeyer, M.E. Walker and C. Weibel: The K-theory of toric varieties, Trans. Amer. Math. Soc. 361 (2009), no. 6, 3325-3341.

[19] G. Cortiñas, C. Haesemeyer, M.E. Walker and C. Weibel: A negative answer to a question of Bass, Proc. Amer. Math. Soc. 139 (4) (2011), 1187-1200.

[20] G. Cortiñas, C. Haesemeyer, M.E. Walker and C. Weibel: K-theory of cones of smooth varieties, J. Alg. Geom. 22 (2013), 13-34.

[21] G. Cortiñas, C. Haesemeyer, and C. Weibel: K-regularity, cdh-fibrant Hochschild homology, and a conjecture of Vorst, J. Am. Math. Soc. 21 (2) (2008), 547-561.

[22] A. J. De Jong: Smoothness, semi-stability and alterations, Publications Mathématiques de l'IHÉS , 83 (1996), 51-93.

[23] V. Drinfeld: DG quotients of DG categories, J. of Algebra, 272 (2004), 5, 643-691. 
[24] P. Du Bois: Complexe de de Rham filtré d'une variété singulière, Bull. Soc. Math. France, 109 (1) (1981), $41-81$.

[25] D. Edidin and W. Graham: Equivariant intersection theory, Invent. Math. 131 (1998), 595-634.

[26] A.I. Efimov: Homotopy finiteness of some DG categories from algebraic geometry, arXiv:1308.0135.

[27] B. Fantechi, E. Mann, F. Nironi: Smooth toric Deligne-Mumford stacks, J. Reine Angew. Math. 648 (2010), $201-244$.

[28] Z. Flores: G-groups of Cohen-Macaulay Rings with n-Cluster Tilting Objects, arXiv:1308.0135.

[29] J. Forgaty, F. Kirwan and D. Mumford: Geometric Invariant Theory, Third Enlarged Edition, SpringerVerlag, 1994.

[30] W. Fulton: Rational equivalence on singular varieties, Publications Mathématiques de l'IHÉS 45 (1975), $147-165$.

[31] W. Fulton: Intersection theory, Second Edition, Springer-Verlag New York, 1998.

[32] W. Fulton and S. Lang: Riemann-Roch Algebra, Springer-Verlag Berlin, 1985.

[33] H. Gillet: K-Theory and Intersection Theory, In: Friedlander E., Grayson D. (eds) Handbook of K-Theory (2005), Springer-Verlag Berlin, 235-293.

[34] G. Gonzalez-Sprinberg and J.L. Verdier: Construction géométrique de la correspondance de McKay, J. Amer. Math. Soc. 14 (2001), 535-554.

[35] S. Gratz, G. Stevenson: Homotopy invariants of singularity categories arXiv:1803.06144

[36] A. Grothendieck, Eléments de géometrie algébraique IV: Étude locale des schémas et des morphismes de schémas, Première partie (EGA 4), Publications Mathématiques de l'IHÉS , 20 (1964), p. 5-259.

[37] I. D. Gubeladze: Anderson's conjecture and the maximal class of monoids over which projective modules are free, Math. USSR Sb. 63 (1989), no. 1, 165-180.

[38] I. D. Gubeladze: Toric varieties with huge Grothendieck group, Adv. Math. 186 (2004), no. 1, $117-124$.

[39] R. Hartshorne: Algebraic geometry, Graduate Texts in Mathematics 52, Springer-Verlag, New-York, (1977).

[40] J. Herzog, E. Marcos and R. Waldi: On the Grothendieck group of a quotient singularity defined by a finite abelian group, J. Algebra 149 (1992), 122-138.

[41] H. Holm: K-groups for rings of finite Cohen-Macaulay type, Forum Math. 27 (2015), no. 4, $2413-2452$.

[42] R. Joshua, A. Krishna: Higher K-theory of toric stacks, Ann. Sc. Norm. Super. Pisa Cl. Sci. (5) 14 (2015), no. 4, 1189-1229.

[43] A. Kuznetsov: Derived categories of families of sextic del Pezzo surfaces, arXiv:1708.00522.

[44] A. Krishna, V. Srinivas: Zero-cycles and K-theory on normal surfaces, Ann. of Math. (2) 156 (2002), no. $1,155-195$.

[45] M. Levine: Modules of finite length and K-groups of surfaces singularities, Compositio Math. 59 (1986), 21-40.

[46] M. Levine: Localization on singular varieties, Invent. Math. 91 (1988), 423-464.

[47] M. Levine, C. Weibel: Zero cycles and complete intersections on singular varieties, J. Reine Angew. Math. 359 (1985), 106-120.

[48] V. Lunts and D. Orlov: Uniqueness of enhancements for triangulated categories, J. Amer. Math. Soc. 23 (2010), 853--908.

[49] E. Marcos: Grothendieck Groups of Quotient Singularities, Trans. Amer. Math. Soc. 332 (1992), no. 1, 93-119.

[50] A. Massey: The KH-theory of complete simplicial toric varieties and the algebraic K-theory of weighted projective spaces, J. Pure Appl. Algebra 217 (2013), no. 11, 2108-2116. 
[51] M.P. Murthy: Vector bundles over affine surfaces birationally equivalent to a ruled surface, Ann. of Math. (2) 89, 1969, 242-253.

[52] V. Navkal: K'-theory of a local ring of finite Cohen-Macaulay type, J. K-Theory 12 (2013), no. 3, 405-432.

[53] A. Nijenhuis, H. Wilf: Representations of integers by linear forms in nonnegative integers, J. Number Theory $4197298-106$.

[54] F. Oort: A construction of generalized Jacobian varieties by group extensions, Math. Ann. $1471962277-$ 286.

[55] D. Orlov: Projective Bundles, Monoidal Transformations, and Derived Categories of Coherent Sheaves, Russian Acad. Sci. Izv. Math. (1993), no. 41 (133), 133-141.

[56] D. Orlov: Triangulated categories of singularities and D-branes in Landau-Ginzburg models, Proc. Steklov Inst. Math. (2004), no. 3 (246), 227-248.

[57] D. Orlov: Formal completions and idempotent completions of triangulated categories of singularities, Adv. Math. 226 (2011), no. 1, 206-217.

[58] D. Quillen: Higher algebraic K-theory I, Higher K-theories, Springer Lect. Notes Math. 341 (1973), 85-147.

[59] P.C. Roberts, V.Srinivas: Modules of finite length and finite projective dimension, Invent. Math. 151 (2003), no. 1, 1-27.

[60] M. Schlichting: A note on K-theory and triangulated categories, Invent. Math. 150 (2002), 111-116.

[61] M. Schlichting: Negative K-theory of derived categories, Math. Z. 253 (2006), no. 1, 97-134.

[62] M. Schlichting: Higher algebraic K-theory, Topics in Algebraic and Topological K-Theory, Lect. Notes in Math. 2008 (2011), Springer, Berlin, 167-241.

[63] O. M. Schnürer: Six operations on dg enhancements of derived categories of sheaves, Selecta Math. (N.S.) 24 (2018), no. 3, 1805-1911.

[64] V. Srinivas: Modules of finite length and Chow groups of surfaces with rational double points, Ill. J. Math. 31(1986), 36-61.

[65] V. Srinivas: $K_{1}$ of the cone over a curve, J. Reine Angew. Math. 381 (1987), 37-50.

[66] V.Srinivas: Algebraic cycles on singular varieties Proceedings of the International Congress of Mathematicians. Volume II, 603-623, Hindustan Book Agency, New Delhi, 2010.

[67] A. Suslin: On the K-theory of local fields, Proceedings of the Luminy conference on algebraic K-theory (Luminy, 1983). J. Pure Appl. Algebra 34 (1984), no. 2-3, 301-318.

[68] A. Suslin and V. Voevodsky: Bloch-Kato conjecture and motivic cohomology with finite coefficients, in The Arithmetic and Geometry of Algebraic Cycles (Banff, AB, 1998). NATO Sci. Ser. C Math. Phys. Sci. 548 (2000), 117-189, Kluwer Acad. Publ., Dordrecht.

[69] G. Tabuada: Algebraic K-theory with coefficients of cyclic quotient singularities, C. R. Math. Acad. Sci. Paris 354 (2016), no. 5, 449-452.

[70] G. Tabuada: $\mathbb{A}^{1}$-homotopy invariance of algebraic $K$-theory with coefficients and du Val singularities, Ann. K-Theory 2 (2017), no. 1, 1-25.

[71] R.W. Thomason: Algebraic K-theory of group scheme actions, Annals of Math. Study 113 (1987), 539 - 563.

[72] R.W. Thomason: The classification of triangulated subcategories, Compositio Math. 105 (1997), no. 1, $1-27$.

[73] R.W. Thomason and T. Trobaugh: Higher Algebraic K-Theory of Schemes and of Derived Categories, The Grothendieck Festschrift Volume III, Volume 88, Progr. Math. Birkhäuser Boston, 1990, 247-435.

[74] J.L. Verdier: Categories derivées, SGA 4 1/2, Lecture Notes in Math., v. 569, Springer-Verlag, 1977.

[75] A. Vistoli: Higher equivariant K-Theory for finite group actions, Duke Math. J. 63 (1991), no. 2, 399-419.

[76] C. Weibel: The negative K-theory of normal surfaces, Duke Math. J. 108 (2001), no. 1, 1-35. 
[77] J. Wlodarczyk: Toroidal varieties and the weak factorization theorem, Invent. Math. 154(2):223-331, 2003.

[78] Y. Yoshino: Cohen-Macaulay modules over Cohen-Macaulay rings, London Mathematical Society Lecture Note Series, 146. Cambridge University Press, Cambridge, 1990. 